\title{
Meanders and eddy formation by a buoyant coastal current flowing over a sloping topography
}

\author{
Laura Cimoli ${ }^{1,3}$, Alexandre Stegner ${ }^{2}$, and Guillaume Roullet ${ }^{3}$ \\ ${ }^{1}$ University of Brest, CNRS, IRD, Ifremer, Laboratoire d'Océanographie Physique et Spatiale, IUEM, Brest, France \\ ${ }^{2}$ Laboratoire de Météorologie Dynamique, CNRS/École Polytechnique/Université Paris-Saclay, Palaiseau, France \\ ${ }^{3}$ Department of Physics, University of Oxford, Oxford, UK
}

Correspondence to: Laura Cimoli (laura.cimoli@physics.ox.ac.uk)

Received: 18 March 2017 - Discussion started: 18 April 2017

Revised: 11 July 2017 - Accepted: 4 August 2017 - Published: 14 November 2017

\begin{abstract}
This study investigates the linear and non-linear instability of a buoyant coastal current flowing along a sloping topography. In fact, the bathymetry strongly impacts the formation of meanders or eddies and leads to different dynamical regimes that can both enhance or prevent the crossshore transport. We use the Regional Ocean Modeling System (ROMS) to run simulations in an idealized channel configuration, using a fixed coastal current structure and testing its unstable evolution for various depths and topographic slopes. The experiments are integrated beyond the linear stage of the instability, since our focus is on the non-linear end state, namely the formation of coastal eddies or meanders, to classify the dynamical regimes. We find three nonlinear end states, whose properties cannot be deduced solely from the linear instability analysis. They correspond to a quasi-stable coastal current, the propagation of coastal meanders, and the formation of coherent eddies. We show that the topographic parameter $T_{\mathrm{p}}$, defined as the ratio of the topographic Rossby wave speed over the current speed, plays a key role in controlling the amplitude of the unstable crossshore perturbations. This result emphasizes the limitations of linear stability analysis to predict the formation of coastal eddies, because it does not account for the non-linear saturation of the cross-shore perturbations, which is predominant for large negative $T_{\mathrm{p}}$ values. We show that a second dimensionless parameter, the vertical aspect ratio $\gamma$, controls the transition from meanders to coherent eddies.

We suggest the use of the parameter space $\left(T_{\mathrm{p}}, \gamma\right)$ to describe the emergence of coastal eddies or meanders from an
\end{abstract}

unstable buoyant current. By knowing the values of $T_{\mathrm{p}}$ and $\gamma$ for an observed flow, which can be calculated from hydrological sections, we can identify which non-linear end state characterizes that flow - namely if it is quasi-stable, meanders, or forms eddies.

\section{Introduction}

Coastal currents can act either as a source of coherent eddies or as a dynamical barrier to the offshore redistribution of coastal waters, thus controlling the cross-shelf transport in a local or regional circulation. When these currents are unstable, large meanders grow and can lead to the formation of mesoscale eddies that capture and transport water masses towards the open sea over large stretches of the coastline. Conversely, stable coastal currents enhance the alongshore transport and strongly reduce the cross-shelf transport. Hence, the formation and the propagation of coastal eddies across the coastal shelf plays a significant role in the local mixing of biogeochemical properties, in the dispersion of pollutants, and in the redistribution of nutrient-rich coastal waters toward the oligotrophic open sea (Riandey et al., 2005).

On the one hand, many coastal currents such as the Algerian Current (Millot, 1999; Obaton et al., 2000; Puillat et al., 2002), the West Greenland Current (Eden and Boning, 2002; Pickart et al., 2005; Hátún et al., 2007), and the Norwegian Coastal Current (Björk et al., 2001) shed coherent 
and long-lived eddies off the coast. On the other hand, some coastal flows, such as the Bransfield Current (Savidge and Amft, 2009; Sangrá et al., 2011; Poulin et al., 2014) or the Northern Current in the western Mediterranean Sea (Sammari et al., 1995; Millot, 1999; Birol et al., 2010), appear to be quasi-stationary along the shelf slope. Hence, the impact of a sloping bathymetry on the development and the evolution of meanders and eddies has been the topic of several studies. The first studies were devoted to the linear stability of coastal flows, while the more recent numerical simulations focus on the non-linear formation of meso- and submesoscale eddies from shelf/slope density fronts or currents (Pennel et al., 2012; Stewart and Thompson, 2013; Gula et al., 2014).

There exist numerous linear stability analyses of baroclinic currents flowing over sloping topography which are based on layered models (Mysak, 1977; Mysak et al., 1981; Gula and Zeitlin, 2014; Poulin et al., 2014) or continuous stratification (Blumsack and Gierasch, 1972; Mechoso, 1980; Lozier et al., 2002; Lozier and Reed, 2005; Isachsen, 2011). In the framework of quasi-geostrophic (QG) models, both the two-layer model and the continuously stratified Eady model show that when the isopycnals and the topographic slopes tilt in opposite directions, a sloping topography reduces the growth rate of baroclinic modes with respect to a flat bottom case. These idealized stability analyses demonstrate that the central parameter of the problem is not the bottom slope $(s)$, but the ratio of the bottom slope over the isopycnal slope $(\alpha)$, defined as the topographic parameter. This ratio, $T_{0}=s / \alpha$, is, by convention, negative when the shelf and the isopycnals tilt in the opposite sense. Poulin et al. (2014) extend these analyses to a two-layer, shallow-water framework, and show that, as for the QG models, the increase in the bottom slope tends to stabilize the baroclinically unstable modes. They also show that, when the structure of the coastal jet allows for both baroclinic and barotropic instabilities, the stabilization of the baroclinic modes leads to the dominance of the barotropic horizontal shear instability.

Hence, for a geostrophic coastal current, the linear stability analysis predicts that two different regimes of instability with distinct wavelength selection can occur above the shelf bathymetry. When the geostrophic coastal current is controlled by the baroclinic instability, the decrease in the topographic parameter $T_{0}$ (with $T_{0}<0$ ) yields a selection of smaller unstable wavelengths, which can be 2 or 3 times smaller than the one emerging in a flat bottom configuration. However, for more negative values of $T_{0}$, the growth rates of baroclinic modes decreases strongly and the horizontal shear instability becomes dominant. The latter is then weakly affected by the shelf slope and leads to large unstable wavelengths (Poulin et al., 2014).

However, it is well known that the linear stability analysis is limited by its inability to predict the final amplitude of unstable meanders. Furthermore, non-linear processes may lead to larger or smaller structures than the ones predicted by the linear analysis. Moreover, previous studies have shown that a sloping topography has a strong impact on the non-linear saturation of unstable surface flows (Sutyrin, 2001). Experimental studies (using a two-layer stratification) of coastal fronts or coastal currents over linear shelf slopes (Pennel et al., 2012; Geheniau et al., 2017) have shown that there are no large meanders or any eddy detachments when the topographic parameter reaches values below $T_{0} \simeq-3$. This is evidence that the non-linear saturation of the linear instability becomes important when the topographic slopes are comparable to the isopycnal slopes.

The primary goal of this study is to go beyond the linear stability analysis and investigate the non-linear impact of the sloping topography on the formation of coastal eddies, namely whether or not the current generates a significant non-linear cross-shore disturbance. To answer this question, we use an idealized model of a buoyant current with a continuous and non-uniform stratification. Indeed, the effects of a continuous stratification and the relevant set of dynamical parameters that govern the stability of geostrophic currents along a sloping bathymetry are not well established. Unlike standard linear instability analysis, the use of a full non-linear model allows us to discuss the end state of the instability, in other words the various regimes of formation of largescale meanders or coastal eddies. We find that investigating the non-linear evolution shows three possible non-linear end states, while the linear analysis predicts only two types of instability - barotropic and baroclinic. A classification of the various non-linear end states provides a more direct comparison with surface oceanic observations, such as sea surface temperature (SST) images or sea surface height (SSH) maps, where only finite-amplitude perturbations or coherent eddies can be detected.

In Sect. 2, we present the initial state of the coastal current and the main dynamic and topographic parameters of the system. In Sect. 3, we investigate how different values of the bottom slope and depth affect the stability of a surface intensified current. We then show, in Sect. 4, the role played by the topographic parameter in controlling the amplitude of the unstable perturbations. If these perturbations reach a finite amplitude, large meanders or coherent eddies are formed. The characteristics of these final non-linear structures and their corresponding parameter space are given in Sects. 5 and 6. Discussions and conclusions are given in the final Sect. 7.

\section{Numerical model setup}

To investigate the effect of a sloping topography on meanders and eddy formation in a buoyant coastal current, we employ the Agrif version (Penven et al., 2006; Debreu et al., 2011) of the Regional Oceanic Modeling System (ROMS) (Shchepetkin and McWilliams, 2003, 2005). While keeping the model setup idealized, our configuration has been built with a particular observed ocean current system in mind, namely the Bransfield Current in Antarctica. This decision 
stems from our original motivation of extending the analysis made with a two-layer, shallow-water model presented in Poulin et al. (2014) to a continuously stratified, 3-D primitive equations model.

We use a periodic rectangular domain, with $x$ as the alongshore axis, $y$ the cross-shore axis (positive offshore), and $z$ the vertical axis (the model uses terrain-following vertical coordinates). Two distinct grid resolutions are used, a coarse grid resolution $(\mathrm{d} x=\mathrm{d} y=2 \mathrm{~km}$ and $N=32 \sigma$ vertical levels) and a higher-resolution grid $(\mathrm{d} x=\mathrm{d} y=0.6 \mathrm{~km}$ and $N=32 \sigma$ vertical levels). Most of the results presented here come from the low-resolution runs, but we found a good convergence of the higher-resolution simulations for various dynamical regimes identified. The horizontal domain dimensions are $L_{x}=256 \mathrm{~km}$ and $L_{y}=160 \mathrm{~km}$, respectively. This configuration corresponds to a coastal channel with periodic boundary conditions for the along-shore direction, and free slip boundaries at the coast $(y=0)$ and offshore $\left(y=L_{y}\right)$. The effect of the bottom friction is here neglected by setting it to zero, since the focus of this paper is on the inviscid dynamics and a non-zero bottom friction would slow down the current during the initial period of integration.

The initial state consists of a steady geostrophic surface current flowing along an idealized sloping bathymetry (Fig. 1a). The water depth, at a given distance $y$ from the coast, is given by a hyperbolic tangent profile:

$h(y)=H+\Delta h \tanh \left[s\left(y-L_{s}\right) / \Delta h\right]$,

where $H$ is water depth below the maximum current velocity, $\Delta h$ the height amplitude, $L_{s}$ the pivotal distance, and $s$ the maximal shelf slope (Fig. 1a). Throughout this study we keep $L_{s}=10 \mathrm{~km}$ and $\Delta h=600 \mathrm{~m}$ constant, while we vary both $H$ and $s$. This profile has been often used as a generic bathymetry in previous works (Lozier et al., 2002; Lozier and Reed, 2005; Poulin and Flierl, 2005; Stewart and Thompson, 2013), and according to Poulin et al. (2014), the hyperbolic tangent profile fits remarkably well the shelf bathymetry in the Bransfield Strait, from which the numerical setup of this work takes inspiration.

We use a linear equation of state and set salinity to a constant; thus, the density stratification is a function of temperature only and is equal to $\rho=-\rho_{0} \alpha_{\mathrm{T}} T$. The thermal stratification for the unperturbed ocean is defined by the following function:

$T_{\mathrm{oc}}=T_{0}+\frac{N_{0}^{2}}{g \alpha_{\mathrm{T}}} z+\Delta T \tanh \left(z / H_{\mathrm{T}}\right)$,

where $N_{0}\left(\simeq 2.4 \times 10^{-4} \mathrm{~s}^{-1}\right)$ is a minimal Brunt-Väisälä frequency in the deep layer, $\Delta T=2{ }^{\circ} \mathrm{C}$ is the temperature difference between the surface and the deep ocean, $g$ is the gravitational acceleration, and $\alpha_{\mathrm{T}}$ is the thermal expansion coefficient of seawater $\left(\alpha_{\mathrm{T}}=\partial_{\mathrm{T}} \rho / \rho_{0} \simeq 2.8 \times 10^{-4} \mathrm{~K}^{-1}\right)$. Hence, the relative Brunt-Väisälä frequency varies from $N_{\mathrm{s}} / f=40$ at the surface to $N_{0} / f \simeq 2$ at the bottom (Fig. 1b). The typical unperturbed thermocline depth is set here at $H_{\mathrm{T}}=200 \mathrm{~m}$.
The coastal current, in thermal wind balance, is driven by a warm-temperature anomaly above the shelf, which we define as

$$
\begin{aligned}
& T_{\text {an }}(y, z)=\Delta T_{\text {jet }} F(y) G(z), \\
& F(y)=1-\frac{1}{2}\left[1+\tanh \left(\frac{y-D}{L}\right)\right] \tanh ^{2}(1.2 y / D), \\
& G(z)=\exp \left(-z^{2} / 2 H_{\text {jet }}^{2}\right) .
\end{aligned}
$$

The width and the depth of the coastal jet are fixed by $L=10 \mathrm{~km}$ and $H_{\text {jet }}=250 \mathrm{~m}$, respectively. $D$ is the distance to the coast, and in all our analysis we use $D=L=L_{s}=$ $10 \mathrm{~km}$. The temperature difference $\Delta T_{\text {jet }}$ is determined indirectly from the maximum jet velocity $U_{\max }$, obtained upon vertical integration of the thermal wind balance

$\partial_{z} U(y, z)=\frac{g}{f} \partial_{y} \rho=-\frac{\alpha_{\mathrm{T}} \rho_{0}}{f} G(z) \partial_{y} F(y)$,

assuming $U=0$ at $z=H+\Delta h$, i.e. over the flat part of the domain, and where the Coriolis parameter $f=1.26 \times$ $10^{-4} \mathrm{~s}^{-1}$ is constant. The surface velocity $U_{0}(y)=U(y, z=$ 0 ) is then proportional to $\partial_{y} F(y)$, and is thus quite similar to the linear Gaussian jet used by Poulin et al. (2014) to model the Bransfield Current. The velocity section in the vertical plane (Fig. 1) shows a surface-intensified current with very low values in the deep layer below $H_{\text {jet }}=250 \mathrm{~m}$. In the absence of any wind stress forcing there is no reason to consider a velocity shear $\mathrm{d} U / \mathrm{d} z$ at the surface, and we therefore choose a Gaussian law for the vertical profile $G(z)$.

The model solves the primitive equations with a splitexplicit free surface, where short time steps are used to advance the surface elevation and barotropic momentum equation, and a larger time step is used for temperature and baroclinic momentum. We stick to the ROMS philosophy of removing explicit horizontal turbulent closure terms on both temperature (no diffusion) and momentum (no viscosity), and of letting the third-order upstream-biased advection operator handle the necessary dissipation at grid scale. We consider initial value problems with no external forcing, but only an initial white noise added to the velocity field. Consequently, the surface momentum, heat, and freshwater fluxes are set to zero.

\subsection{Dynamic and topographic parameter}

For a flat bottom configuration, the dynamics of the coastal current mainly depend on the Rossby $(R o)$ and the Burger (Bu) numbers:

$$
R o=\frac{U_{\max }}{f L}, \quad B u=\left(\frac{R_{\mathrm{d}}}{L}\right)^{2},
$$

where $R_{\mathrm{d}}$ is the first baroclinic deformation radius. We compute the linear eigenmodes and the corresponding deformation radius $R_{\mathrm{d}}$ of the vertical stratification $\rho(y=D, z)$ at the 

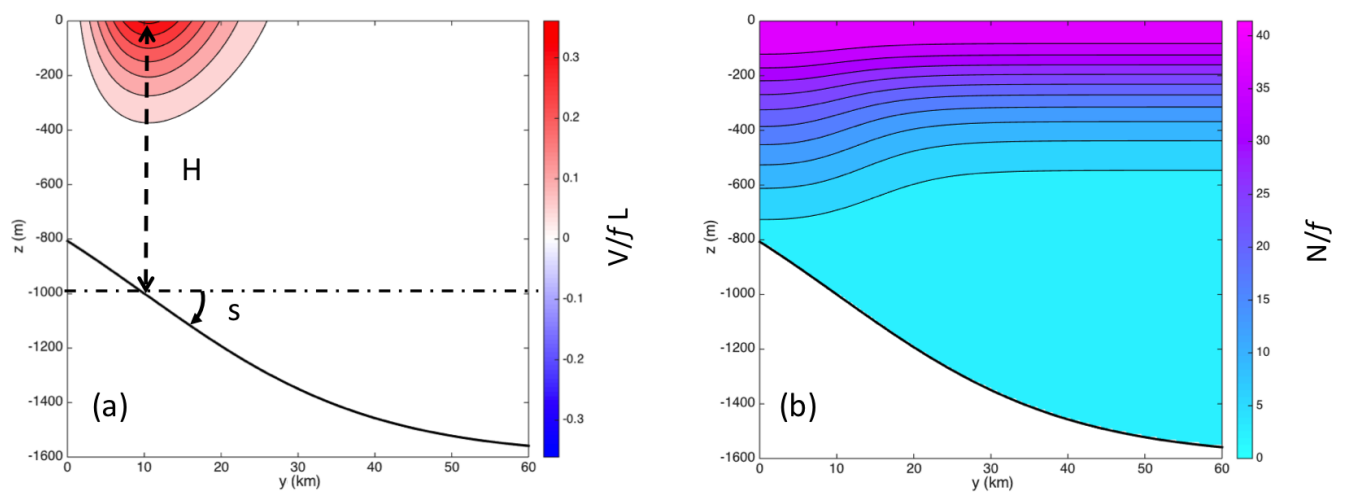

Figure 1. Simplified configuration of a surface coastal current above an hyperbolic tangent bathymetry. The along-shore velocity (a) and the Brunt-Väisälä frequencies (b) are plotted in dimensionless form $(V / f L, N / f)$ and correspond to the configuration where $H=1000 \mathrm{~m}$, $L=10 \mathrm{~km}, s=3 \%, \Delta T_{\mathrm{jet}}=0.8^{\circ} \mathrm{C}, U_{\max }=35 \mathrm{~cm} \mathrm{~s}^{-1}, f=1.26 \times 10^{-4} \mathrm{~s}^{-1}$.

location of the maximum current velocity $U_{\max }$, which is for a depth $H$. The standard eigenmode equation and the typical structure of the first baroclinic mode are given in Appendix A. The typical values for the first baroclinic deformation radius range between $R_{\mathrm{d}} \simeq 5 \mathrm{~km}$ and $R_{\mathrm{d}} \simeq 7 \mathrm{~km}$, leading to small Burger numbers, $B u=0.25-0.5$. Thus, the available potential energy of the initial flow is higher than its kinetic energy.

In this study the intensity of the initial current is kept fixed with a maximum surface velocity $U_{\max }=35 \mathrm{~cm} \mathrm{~s}^{-1}$. The corresponding Rossby number is then $R o=U_{\max } /(f L)=$ 0.25 and the anticyclonic (cyclonic) vorticities maximum values are $\zeta_{\min } / f \simeq-0.35\left(\zeta_{\max } / f \simeq 0.2\right)$. The relative vorticity never goes below the threshold $\zeta / f=-1$ of inertial instability (Knox, 2003; Plougonven and Zeitlin, 2009). Hence, at the first order of approximation, the dynamical evolution of the coastal current satisfies geostrophic balance.

To establish a contact point with two-layer theories, we introduce the vertical aspect ratio parameter:

$\gamma=\frac{H_{\mathrm{jet}}}{H-H_{\mathrm{jet}}}$,

where $H_{\text {jet }}$ is the thickness of the upper layer, encompassing the surface-intensified jet, and $H-H_{\text {jet }}$ is the thickness of the lower layer, almost at rest. This parameter is the close equivalent of the two-layer ratio parameter $\gamma_{2}=H_{1} / H_{2}$ that controls the baroclinic instability in the standard QG Phillips model with unequal layer thicknesses (Phillips, 1954; Pedlosky, 1987; Vallis, 2006). For a flat bottom configuration, the largest growth rates are found when $\gamma_{2}=1$ and the baroclinic growth rate vanishes when $\gamma_{2}$ tends towards zero (Poulin et al., 2014). Hence, if we consider a continuously stratified flow above a flat bottom $(s=0)$ and if we keep $R o$ and $B u$ constant, the geostrophic instabilities of the surface current will then be controlled by the single parameter left, namely $\gamma$.
This is not the case in a sloping topography scenario. In fact, since the current flows above a sloping bathymetry, a key dynamical feature is the interaction of the current with the topographic Rossby waves. In the case of a buoyant coastal current, the topographic Rossby waves propagate in the same direction as the flow. The propagation speed of these waves is proportional to the dimensionless topographic slope $s$. Previous studies, using the continuously stratified Eady model (Blumsack and Gierasch, 1972; Mechoso, 1980; Isachsen, 2011), have shown that the ratio of the bottom slope $(s)$ over the isopycnal slope $(\alpha)$ is the relevant topographic parameter of the problem. However, in our case there is no such definite unique value for the isopycnal slope because isopycnals are bent and so their slopes vary with depth. Therefore, we define a topographic parameter, $T_{\mathrm{p}}$, as the ratio of the characteristic speed of topographic Rossby waves, $U_{\mathrm{TRW}}$, over the maximum speed of the surface current:

$T_{\mathrm{p}}=\frac{U_{\mathrm{TRW}}}{U_{\max }}=-\frac{s f R_{\mathrm{d}}^{2}}{H U_{\max }}$.

The speed $U_{\mathrm{TRW}}=-s f /\left(H k^{2}\right)=-s f R_{\mathrm{d}}^{2} / H$ corresponds here to a characteristic phase speed of topographic Rossby waves, with a typical scale $k R_{\mathrm{d}}=1$. This topographic parameter can also be written in terms of a topographic beta parameter $\beta_{\mathrm{t}}=-s f / H$, as

$T_{\mathrm{p}}=\frac{\beta_{\mathrm{t}} R_{\mathrm{d}}^{2}}{U_{\max }}$.

We will see later that $T_{\mathrm{p}}$, including information both of the topographic slope $(s)$ and the depth $(H)$, is the key parameter that controls the non-linear saturation of the coastal current, namely the formation of meanders and eddies that enhance the cross-shore transport. 


\section{The effect of variable topographic slope $(s)$ and $\operatorname{depth}(H)$}

In this section we present how different slopes and bottom depths can affect the growth rate and the non-linear saturation of the cross-shore perturbations, while keeping all the other parameters and the buoyant current constant.

At the initial stage the coastal current is mainly alongshore; hence we can assume that $V \ll U$, namely the crossshore velocity, $V$, is much smaller than the along-shore velocity, $U$. Therefore, the growth of the cross-shore velocity $V$ is directly proportional to the growth of unstable perturbations that induce a cross-shore transport. Hence, in order to study both the exponential growth and the non-linear amplitude of these perturbations, we calculate at each time step the domain-integrated ratio:

$$
R(t)=2 \frac{\mathrm{KE}_{y}}{\mathrm{KE}}=2 \frac{\left\langle V^{2}\right\rangle}{\left\langle U^{2}+V^{2}\right\rangle},
$$

where the total kinetic energy $\mathrm{KE}$ is the sum of the crossshore $\left(\mathrm{KE}_{y}\right)$ and the along-shore $\left(\mathrm{KE}_{x}\right)$ contributions to the kinetic energy, and \langle\rangle is the domain integration. The factor of 2 in Eq. (9) has been inserted so that the ratio on the lefthand side approaches 1 as the along-shore and cross-shore contributions to the KE approach parity.

Various metrics could be used to quantify the departure from the initial coastal current. We note here that, while this analysis does not take into account any anisotropic perturbation directed in the along-shore direction, we have checked that this does not change qualitatively the results presented in this study. Moreover, the focus here is on the generation of cross-shore transport by an unstable coastal current, as opposed to a stable along-shore flow that reduces the transport of water offshore. Therefore, we chose this metric to put emphasis on the cross-shore perturbations that may break the along-shore jet and lead to the formation of large meanders or coherent eddies.

The temporal evolution of the ratio in Eq. (9) is plotted in Fig. 2, which shows that the cross-shore contribution of the kinetic energy is, at the initial stage, at least 1 order of magnitude smaller than the along-shore one: $\mathrm{KE}_{y} \ll \mathrm{KE}_{x}(t=$ $0) \simeq \operatorname{KE}(t=0)$. These weak cross-shore motions are due to the initial white noise introduced in the numerical simulations. This initial noise is added only on the velocity field, hence the geostrophic adjustment of this initial unbalanced field leads to a decay of $\mathrm{KE}_{y} / \mathrm{KE}$ during the first days of the simulations. Then, an exponential growth of the above ratio occurs due to the linear instability of the coastal current. As long as the amplitude of the unstable perturbations remains small (i.e. $\mathrm{KE}_{y} \ll \mathrm{KE}$ ), we can quantify the mean growth rates of the instability from the log-linear plot. If we assume that the amplitude of the most unstable mode is proportional to the cross-shore velocity, we can deduce the maximal unstable growth rate $\sigma_{\mathrm{m}}$ from the slopes of the log-linear plot, as shown from the solid lines in Fig. 2b. The slope re- turns the exponential growth $2 \sigma_{\mathrm{m}}$ of $\mathrm{KE}_{y}$. When the unstable currents start to form large meanders or when the detachment of coherent eddies occurs, an equipartition between the cross-shore and the along-shore contributions to the kinetic energy is reached, regardless of the intensity of the eddies. Consequently, the non-linear saturation parameter, namely the temporal maximum of the ratio $R(t)$,

$\varepsilon=\max (R(t))=\max \left(2 \mathrm{KE}_{y} / \mathrm{KE}\right)$,

tends to a value close to unity. We stress here that what we call the non-linear saturation parameter returns only the saturation of the cross-shore perturbations, and does not take into account the along-shore perturbations (which never reach finite-amplitude values). Similar analyses were performed by Pennel et al. (2012) and Geheniau et al. (2017) using the surface kinetic energy because only the surface velocity field, derived with a good accuracy from particle image velocimetry (PIV), is available in laboratory experiments.

We now present the impact that a variable $s$ and/or $H$ have on the growth rate of the instabilities of a buoyant current.

Figure 2a shows that, while keeping a constant water depth $H$, the unstable growth rate decreases as we increase the bathymetric slope. This is expected, since previous studies have shown that a steep shelf slope reduces the unstable growth of baroclinic modes - both with a two-layer model. (Mysak, 1977; Mechoso, 1980; Poulin et al., 2014; Gula and Zeitlin, 2014) and the continuously stratified Eady model (Blumsack and Gierasch, 1972; Mechoso, 1980; Isachsen, 2011)). However, these linear stability analyses can predict neither the non-linear evolution of the unstable perturbations nor the final state of the system. It is only recently that laboratory experiments with a two-layer stratification (Geheniau et al., 2017), have shown that the level of the non-linear saturation (i.e. the saturation parameter $\varepsilon$ ) could be significantly smaller if the topographic parameter $T_{0}=s / \alpha<0$ reaches finite values, namely if the shelf slope is steep enough. Here, we confirm this behaviour for a continuously stratified coastal current. According to Fig. 2a, the saturation parameter (solid circles) decreases from $\varepsilon=0.74$ to $\varepsilon=0.45$ and $\varepsilon=0.034$ when the shelf slope increases from $s=3 \%$, to $s=4 \%$ and $s=5 \%$, respectively.

Now, we analyse the cases of a constant slope $s$ and a variable $H$. For the flat bottom configuration $(s=0)$, when the water depth $H$ increases, the growth rate $\sigma_{\mathrm{m}}$ decreases (Fig. 2b). For the simplified two-layer QG Phillips model (Pedlosky, 1987; Vallis, 2006), it is well known that the growth rates of baroclinic perturbations are mainly controlled by the vertical aspect ratio parameter $\gamma_{2}=H_{1} / H_{2}$. The largest growth rates are found when $\gamma_{2}=1$. When $\gamma_{2}$ is reduced, say by increasing the lower-layer thickness $\mathrm{H}_{2}$, both the growth rate and the most unstable wavenumber decrease. We found a similar behaviour for the continuously stratified configuration. Keeping a constant jet depth $H_{\text {jet }}$ and increasing the bottom depth $H$ yields a slower instability of the surface coastal current. Nevertheless, we found that with 

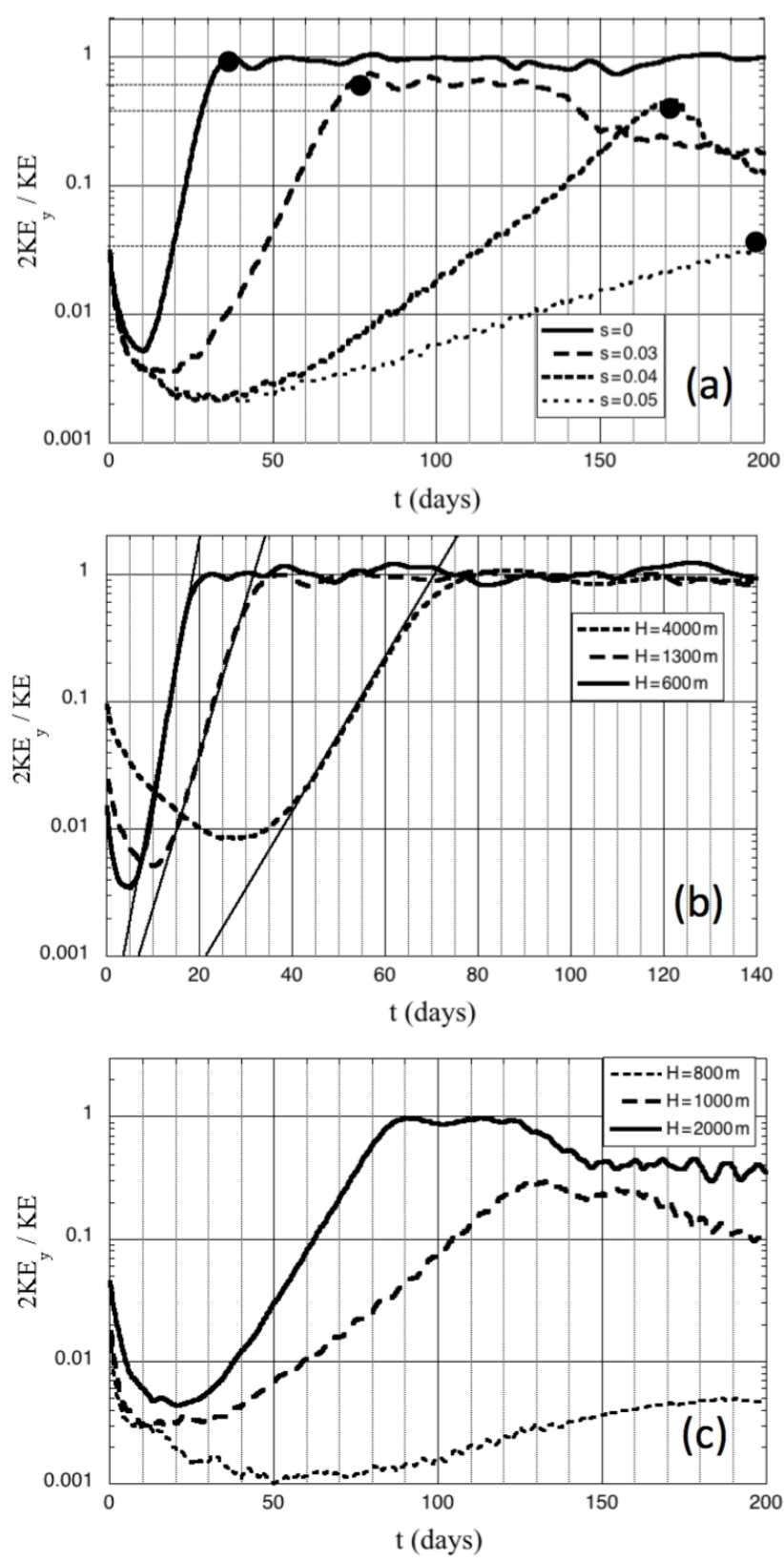

Figure 2. Time evolution of $R(t)=2 \mathrm{KE}_{y} / \mathrm{KE}$. In the upper panel (a) the depth $H=1300 \mathrm{~m}$ is kept constant while the shelf slope varies: $s=0,3,4,5 \%$. The central panel (b) corresponds to the flat bottom configuration ( $s=0$ ) for various depth $H=600,1300$, $4000 \mathrm{~m}$. In the bottom panel (c) the shelf slope $s=3 \%$ is kept constant while the depth varies: $H=800,1000,2000 \mathrm{~m}$.

a flat bottom the unstable perturbations always reach a finite amplitude $(\varepsilon \simeq 1)$.

A similar agreement with the two-layer case is found when we vary the water depth above a sloping bathymetry: as we reduce $H$, we get a stronger stabilization of the surface current. According to Fig. $2 \mathrm{c}$, both the linear growth rate $\sigma_{\mathrm{m}}$ and the non-linear saturation parameter $\varepsilon$ decrease when the wa- ter depth $H$ is decreased while keeping the bottom slope constant at $s=3 \%$. Indeed, for this specific bottom slope, when $H=2000 \mathrm{~m}$ the unstable cross-shore perturbations lead to finite meanders or coastal eddies $(\varepsilon \simeq 1)$ while only very weak cross-shore fluctuations could grow $(\varepsilon \simeq 0.05)$ when $H=800 \mathrm{~m}$.

This initial set of results suggests that both the bottom slope and the water depth have a strong impact on the nonlinear stabilization of the along-shore current. The impact of the sloping bathymetry is increased when the water depth is reduced, and inversely for very large water depths. Extrapolating this to the case of an infinitely deep ocean, we could expect to reduce or even cancel the impact of the bottom slope. The combined effect of variables $s$ and $H$ is described in the following section, where we analyse different quantities with respect to the topographic parameter, which provides a useful re-scaling of the wide range of cases that we have studied (see Table 1).

\section{The role of the topographic parameter $T_{p}$}

In order to quantify more precisely the influence of a sloping bathymetry on the stability of the coastal current, we plot in Fig. 3a the dimensionless growth rates as a function of the topographic parameter $T_{\mathrm{p}}$. Each experiment in Fig. 3 is labelled with either $s$ or $H$, indicating that the companion parameter ( $H$ or $s$, respectively) is varied. Hence, the points with $H=950 \mathrm{~m}$ span $s$ in [0 $4 \%$, which corresponds to $T_{\mathrm{p}}$ in $\left[\begin{array}{ll}-0.5 & 0\end{array}\right]$ (see Table 1).

Figure 3 a shows that for negative values below $T_{\mathrm{p}} \simeq-0.3$, the growth rates of the unstable perturbations decrease towards a relatively small value $\sigma_{\mathrm{m}} R_{\mathrm{d}} / V_{\max } \simeq 10^{-3}$, which is 1 order of magnitude smaller than unstable growth rates in the flat bottom cases $\left(T_{\mathrm{p}}=0\right)$. However, for intermediate values of the topographic parameter $\left(-0.3<T_{\mathrm{p}}<0\right)$, two distinct branches are visible in Fig. 3a. When $T_{\mathrm{p}}$ decreases, the growth rate of the upper branch decreases linearly with $T_{\mathrm{p}}$, whereas the growth rate of the lower branch slightly increases with decreasing $T_{\mathrm{p}}$ until it merges with the upper branch. This behaviour suggests the possible existence of two distinct modes of instability for the same value of $T_{\mathrm{p}}$.

As was shown in Poulin et al. (2014), an efficient way to identify distinct unstable modes is to study the evolution of the most unstable wavelength $\lambda_{\mathrm{m}}$ (or wavenumber $k_{\mathrm{m}}$, where $\left.k_{\mathrm{m}}=2 \pi / \lambda_{\mathrm{m}}\right)$ as a function of the relative slope parameter, which is $T_{\mathrm{p}}$ in our continuously stratified case.

In order to estimate the most unstable wavenumber, we perform, as in Pennel et al. (2012), a Fourier decomposition of the cross-shore velocity at the surface $(z=0)$. We then select the unstable mode $k_{\mathrm{m}}$ that both follows an exponential growth and reaches the highest amplitude. In a few cases, a first mode grows and saturates at a given amplitude while a second one, having similar growth rate, saturates at a higher level after a while. For these specific cases we plot the two 
Table 1. Parameter space of the experiments performed with a $2 \mathrm{~km}$ grid resolution, where $s$ is the topographic slope (\%), $H$ the water depth below the maximum current velocity $(\mathrm{m}),\left|T_{\mathrm{p}}\right|$ is the absolute value of the topographic parameter, $\gamma$ is the vertical aspect ratio, $\sigma_{\mathrm{m}} R_{\mathrm{d}} / V_{\mathrm{max}}$ is the dimensionless growth rate, $\varepsilon$ is the non-linear saturation parameter, $k_{\mathrm{m}} R_{\mathrm{d}}$ is the most unstable wavenumber, and $\mu_{0}$ the averaged conversion rate ratio.

\begin{tabular}{|c|c|c|c|c|c|c|c|c|}
\hline$s(\%)$ & $H$ & $\left|T_{\mathrm{p}}\right|$ & $\gamma$ & $\sigma_{\mathrm{m}} R_{\mathrm{d}} / V_{\max }$ & $\varepsilon$ & $k_{\mathrm{m}} R_{\mathrm{d}}$ & $\mu_{0}$ & Regime \\
\hline 0 & 950 & 0.00 & 0.36 & 0.030 & 1.11 & 0.71 & 1.43 & eddies \\
\hline 0 & 1300 & 0.00 & 0.24 & 0.027 & 1.06 & 0.46 & 1.26 & eddies \\
\hline 0.5 & 950 & 0.06 & 0.36 & 0.029 & 1.35 & 0.85 & 1.73 & eddies \\
\hline 0.5 & 1300 & 0.05 & 0.24 & 0.025 & 1.28 & 0.76 & 1.42 & eddies \\
\hline 1 & 950 & 0.13 & 0.36 & 0.023 & 1.14 & 0.85 & 1.61 & eddies \\
\hline 1 & 1300 & 0.11 & 0.24 & 0.021 & 1.27 & 0.91 & 1.44 & eddies \\
\hline 2 & 950 & 0.25 & 0.36 & 0.010 & 0.60 & 0.98 & 1.51 & meanders \\
\hline 2 & 1300 & 0.21 & 0.24 & 0.014 & 0.95 & 0.91 & 1.32 & meanders \\
\hline 3 & 600 & 0.44 & 0.71 & 0.001 & 0.05 & 0.24 & 0.10 & ASC \\
\hline 3 & 800 & 0.41 & 0.45 & 0.002 & 0.01 & 0.41 & 0.11 & ASC \\
\hline 3 & 950 & 0.38 & 0.36 & 0.002 & 0.19 & 1.30 & 4.32 & meanders \\
\hline 3 & 1000 & 0.37 & 0.33 & 0.005 & 0.29 & 1.16 & 2.12 & meanders \\
\hline 3 & 1100 & 0.35 & 0.29 & 0.008 & 0.63 & 1.18 & 2.27 & meanders \\
\hline 3 & 1300 & 0.32 & 0.24 & 0.012 & 0.74 & 1.05 & 1.44 & meanders \\
\hline 3 & 2000 & 0.24 & 0.14 & 0.011 & 0.97 & 0.97 & 1.43 & meanders \\
\hline 3 & 2500 & 0.20 & 0.11 & 0.010 & 1.11 & 0.99 & 1.06 & meanders \\
\hline 3 & 4000 & 0.13 & 0.07 & 0.005 & 0.88 & 0.52 & 0.45 & eddies \\
\hline 3 & 6000 & 0.09 & 0.04 & 0.008 & 0.93 & 0.53 & 0.52 & eddies \\
\hline 3.5 & 1100 & 0.41 & 0.29 & 0.003 & 0.19 & 1.35 & 1.78 & meanders \\
\hline 4 & 950 & 0.51 & 0.36 & 0.001 & 0.01 & 0.43 & 0.07 & ASC \\
\hline 4 & 1100 & 0.47 & 0.29 & 0.002 & 0.03 & 0.29 & 0.34 & ASC \\
\hline 4 & 1300 & 0.43 & 0.24 & 0.004 & 0.46 & 1.22 & 1.92 & meanders \\
\hline 5 & 1300 & 0.53 & 0.24 & 0.002 & 0.04 & 0.30 & 0.52 & ASC \\
\hline 5 & 2000 & 0.39 & 0.14 & 0.005 & 0.55 & 1.12 & 1.13 & meanders \\
\hline 6 & 1100 & 0.71 & 0.29 & 0.002 & 0.01 & 0.44 & 0.05 & ASC \\
\hline 6 & 1300 & 0.64 & 0.24 & 0.002 & 0.01 & 0.30 & 0.08 & ASC \\
\hline 6 & 2000 & 0.47 & 0.14 & 0.003 & 0.26 & 0.32 & 0.34 & ASC \\
\hline 6 & 2500 & 0.40 & 0.11 & 0.004 & 0.44 & 0.33 & 0.33 & eddies \\
\hline 6 & 3000 & 0.34 & 0.09 & 0.005 & 0.97 & 0.34 & 0.32 & eddies \\
\hline 6 & 5000 & 0.22 & 0.05 & 0.009 & 0.81 & 0.52 & 0.45 & eddies \\
\hline
\end{tabular}

wavenumbers in Fig. 4. We clearly see, in this figure, two distinct branches. For the upper one, the unstable wavenumber $k_{\mathrm{m}} R_{\mathrm{d}}$ increases (i.e. $\lambda_{\mathrm{m}}$ decreases) when the topographic parameter decreases until the limit value $T_{\mathrm{p}} \simeq-0.4$ is reached. Below this value, the upper branch does not exist because its growth rate becomes smaller than that of the lower branch, therefore this mode does not emerge during the time integration. For the lower branch, the most unstable wavenumber is both smaller and much less impacted by the variation of the topographic parameter, suggesting that this mode corresponds to a barotropic mode, as we will soon show.

In order to identify the nature of these two branches we determine the source of kinetic energy of the perturbations for the instability. In this simplified jet configuration, there are basically two source terms (Gula et al., 2015): $\left\langle w^{\prime} b^{\prime}\right\rangle$, the extraction from the potential energy of the jet, and $\left\langle-u^{\prime} v^{\prime} \partial_{y} U\right\rangle$, the extraction from the horizontal shear, where $u^{\prime}=U-\bar{U}$, $v^{\prime}=V-\bar{V}$, and $w^{\prime}=W-\bar{W}$ are the velocity perturbations to the along-shore averaged velocities, and $\bar{U}, \bar{V}$, and $\bar{W}$ are the along-shore averaged velocities. Note that $\bar{U}, \bar{V}$, and $\bar{W}$ vary with time if the jet starts to experience finite-amplitude perturbations. The baroclinic instability is characterized by a dominance of the former source term, whereas horizontal shear instability is characterized by the dominance of the latter. We therefore introduce a conversion rate ratio, which quantifies the extraction of potential energy relative to the extraction of kinetic energy from the horizontal shear:

$\mu(t)=\frac{\left\langle w^{\prime} b^{\prime}\right\rangle}{\left\langle-u^{\prime} v^{\prime} \partial_{y} U\right\rangle}$.

We select the stage of exponential growth of $\mathrm{KE}_{y}$ and compute the mean over this time to get a new dimensionless parameter $\mu_{0}=\overline{\mu(t)}$, which quantifies the baroclinic or barotropic nature of the energy conversion of the linear instability. The nature of the linear instability appears to have a crucial impact on the wavelength selection. We confirm this result in Fig. 4b, where all the points of the upper branch correspond to $\mu_{0}>1$ while the lower branch corresponds to 

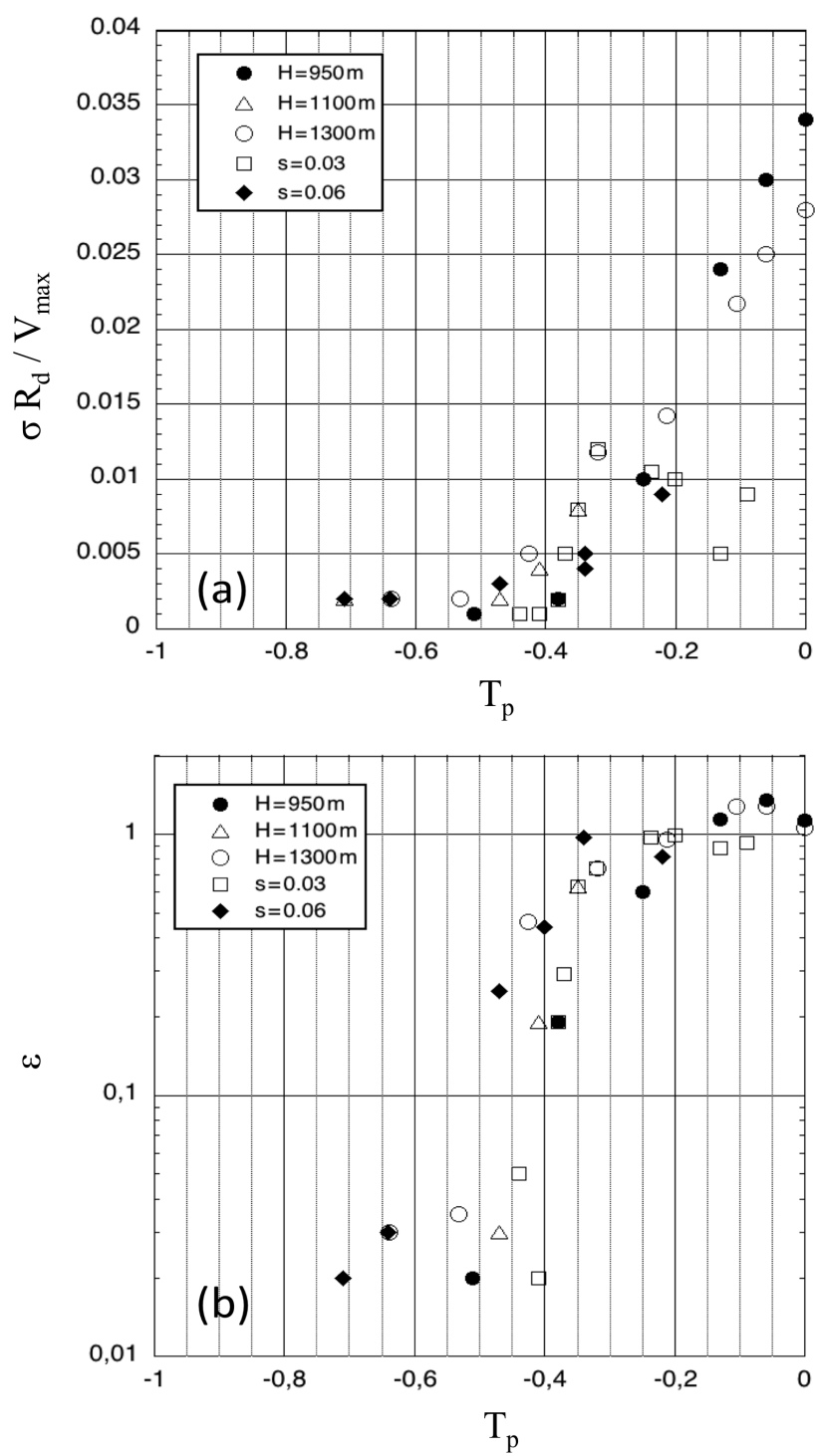

Figure 3. (a) Dimensionless growth rates $\sigma_{\mathrm{m}} R_{\mathrm{d}} / V_{\max }$ and (b) the non-linear saturation parameter $\varepsilon$ as a function of the topographic parameter $T_{\mathrm{p}}$ for the same coastal current $(R o=0.25)$ above various shelves. We consider here a large range of bottom slopes $(s=0-6 \%)$ and water depths $(H=600-5000 \mathrm{~m})$.

$\mu_{0}<1$. Hence, these two separated branches are associated with two distinct mechanisms of instability, namely the baroclinic and the barotropic shear instability.

$T_{\mathrm{p}} \simeq-0.4$ seems to act as a threshold between the baroclinic and barotropic modes, as evident in Fig. 4. Interestingly, we find the same threshold in Fig. 3b, where we plot the non-linear saturation parameter, $\varepsilon$, with respect to $T_{\mathrm{p}}$. The non-linear impact of $T_{\mathrm{p}}$ on the saturation of the unstable cross-shore perturbations is striking: all experiments seem to fit on a single curve. From Fig. $3 b$ we clearly see an abrupt non-linear stabilization of the current when the topographic parameter goes below the threshold value centred around
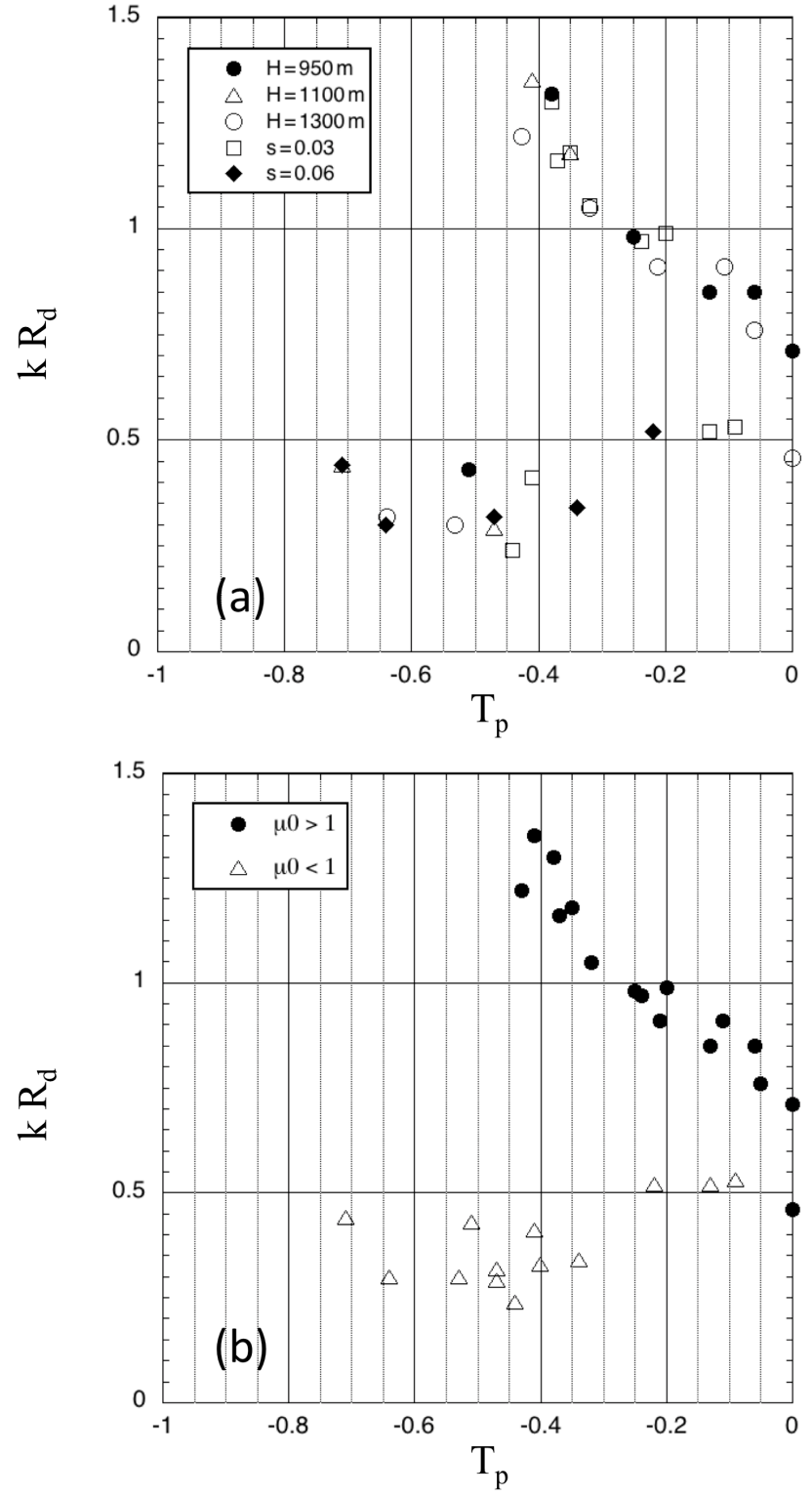

Figure 4. Panel (a): most unstable wavenumbers $k_{\mathrm{m}} R_{\mathrm{d}}$ as a function of the topographic parameter $T_{\mathrm{p}}$ for a wide range of bottom slopes $(s=0-6 \%)$ and water depths $(H=600-5000 \mathrm{~m})$. Panel (b): same as panel (a), but for different values of the mean conversion rate ratio $\left(\mu_{0}\right)$.

$T_{\mathrm{p}}=-0.4 \pm 0.05$. Below this threshold, the stabilization is so strong that the along-shore current, despite being unstable with $\sigma_{\mathrm{m}}>0$, is unable to develop significant cross-shore perturbations. These perturbations saturate at a very weak amplitude and hardly affect the along-shore current. For moderate values $\left(-0.4<T_{\mathrm{p}}<0\right)$ the cross-shore perturbations grow until large meanders or coastal eddies are formed, no matter how large the growth rate is. The fact that all the simulations collapse on a single curve is remarkable. This result 

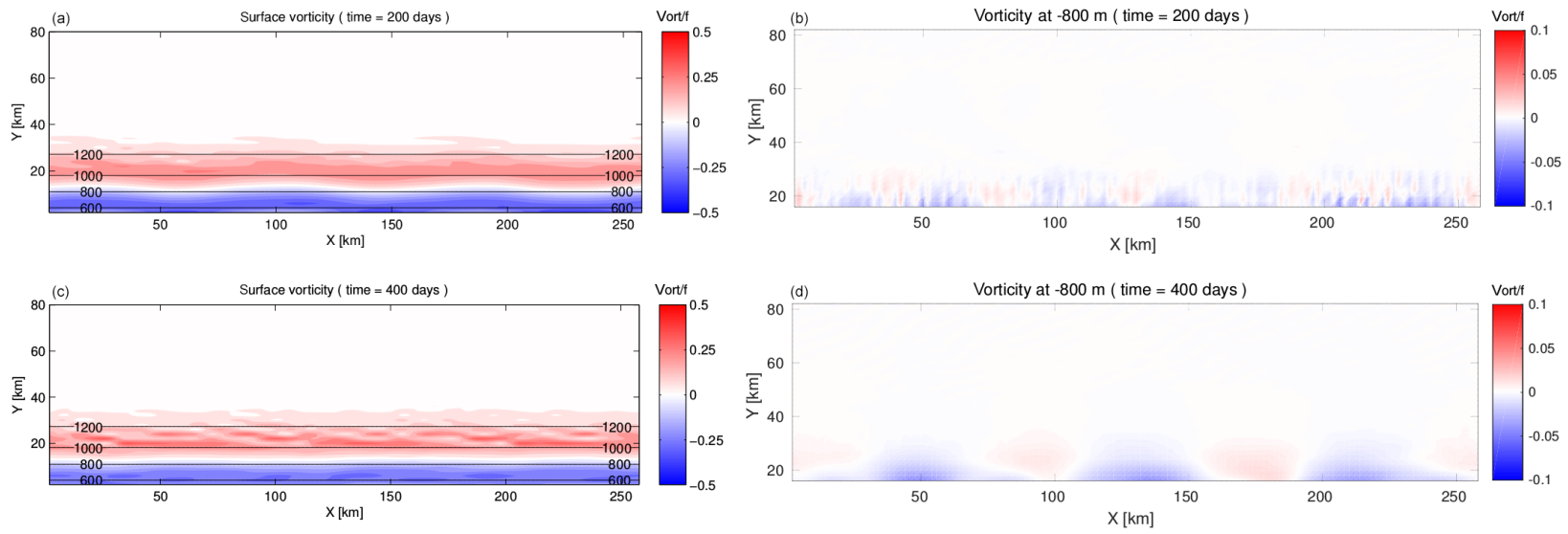

Figure 5. The relative vorticity $(\zeta / f)$ at the surface (left panel) and in the deep layer at $z=-800 \mathrm{~m}$ (right panel) for a shelf slope ( $s=3 \%$, $\left.T_{\mathrm{p}}=-0.41\right)$ when the aspect ratio parameter is $\gamma \simeq 0.45(H=800 \mathrm{~m})$ and the vortex Rossby number is $R o=0.25$. The colour bar of the lower layer is magnified by a factor of 5 in comparison with the upper layer. Panels (a, b) and (c, d) correspond to $t=200$ days and $t=400$ days, respectively.

shows that the topographic parameter $T_{\mathrm{p}}$ is the main parameter that controls the non-linear cross-shore instability.

We now present a possible interpretation on why $T_{\mathrm{p}}=$ -0.4 is the critical value and what changes dynamically at this transition point to explain this result. During the linear stage of the instability, the perturbation grows by extracting energy from the mean flow. When the perturbation becomes large enough, non-linear interactions cause energy to be transferred between modes. Energy transfer is then achieved by the terms of the form $\left\langle-u^{\prime} v^{\prime} \partial_{y} u^{\prime}\right\rangle$, namely cubic terms in the perturbations, as opposed to the quadratic terms, either $\left\langle w^{\prime} b^{\prime}\right\rangle$ or $\left\langle-u^{\prime} v^{\prime} \partial_{y} U\right\rangle$. This non-linear transfer is responsible for the energy cascade in the turbulent regime. If it is dominant then the flow becomes turbulent. The presence of a strong beta effect is known to prevent these non-linear interactions and maintain the flow within a wave regime, dominated by Rossby waves (planetary Rossby waves in the case of planetary beta effect and topographic Rossby waves in the present case). The selection between the wave regime and the turbulent regime depends on the size of the eddies with respect to the Rhines scale (Williams and Kelsall, 2015). Usually the Rhines scale is defined in the context of turbulent flow with $L_{\mathrm{R}}=\sqrt{2 U_{\mathrm{rms}} / \beta}$, where $U_{\mathrm{rms}}$ characterizes the intensity of the eddies. Here we propose to substitute $2 U_{\text {rms }}$ with $U_{\max }$, the jet speed. The rationale is that if eddies form, their $U_{\mathrm{rms}} \sim U_{\mathrm{max}}$. This gives for the Rhines scale $L_{R}=\sqrt{U_{\max } / \beta_{\mathrm{T}}}$, where $\beta_{\mathrm{T}}$ is here the topographic beta parameter. The typical eddy radius is $R_{\mathrm{e}}=\lambda / 4$, where $\lambda$ is the wavelength of the unstable mode. Using the approximation $k R_{\mathrm{d}} \sim 1$ (Fig. 3), we have $R_{\mathrm{e}}=\pi R_{\mathrm{d}} / 2$. Thus, $T_{\mathrm{p}}$ can be rewritten as

$T_{\mathrm{p}}=-\left(\frac{2}{\pi}\right)^{2}\left(\frac{R_{\mathrm{e}}}{L_{\mathrm{R}}}\right)^{2}$.
The discussion on $R_{\mathrm{e}} / L_{\mathrm{R}}$ can now be cast in terms of $T_{\mathrm{p}}$, with a threshold between the wave and the turbulent regimes of $4 / \pi^{2} \simeq 0.4$. For $R_{\mathrm{e}} / L_{\mathrm{R}}<1$, corresponding to $T_{\mathrm{p}}>-0.4$, non-linear interactions can become the leadingorder term in the energy budget. For $R_{\mathrm{e}}>L_{\mathrm{R}}$, corresponding to $T_{\mathrm{p}}<-0.4$, the generated eddies are too large compared to the Rhines scale, preventing the non-linear energy transfer from becoming dominant. The unstable waves stop growing and the flow remains in a wavy regime.

However, by using the topographic parameter alone we are not able to distinguish between different non-linear end states when $-0.4<T_{\mathrm{p}}<0$. Indeed, Fig. 4 has shown that at least two distinct instabilities could occur for the same value of $T_{\mathrm{p}}$, which means that different final states of the flow are possible. This implies that the topographic parameter is not the only dimensionless parameter that controls the unstable regimes of the coastal current above the shelf. We will see shortly that the vertical aspect ratio $\gamma=H_{\text {jet }} /\left(H-H_{\text {jet }}\right)$ is the second parameter to be taken into account.

We present in the next two sections the characteristics and parameter space of the different end states identifiable from the non-linear analysis.

\section{Quasi-stable, along-shore current (ASC)}

We have shown in Figs. $3 \mathrm{~b}$ and 4 that for $T_{\mathrm{p}}<-0.4$ the baroclinic mode is dampened and the non-linear saturation parameter $(\varepsilon \leqslant 0.1)$ reaches values at least 1 order of magnitude smaller than for $T_{\mathrm{p}}>-0.4$. This corresponds to a regime in which the buoyant current is quasi-stable, as evidenced from the relative vorticity (Fig. 5).

From a linear stability perspective, the jet is unstable: waves grow spontaneously from random perturbations, though always very slowly compared to the other regimes. 

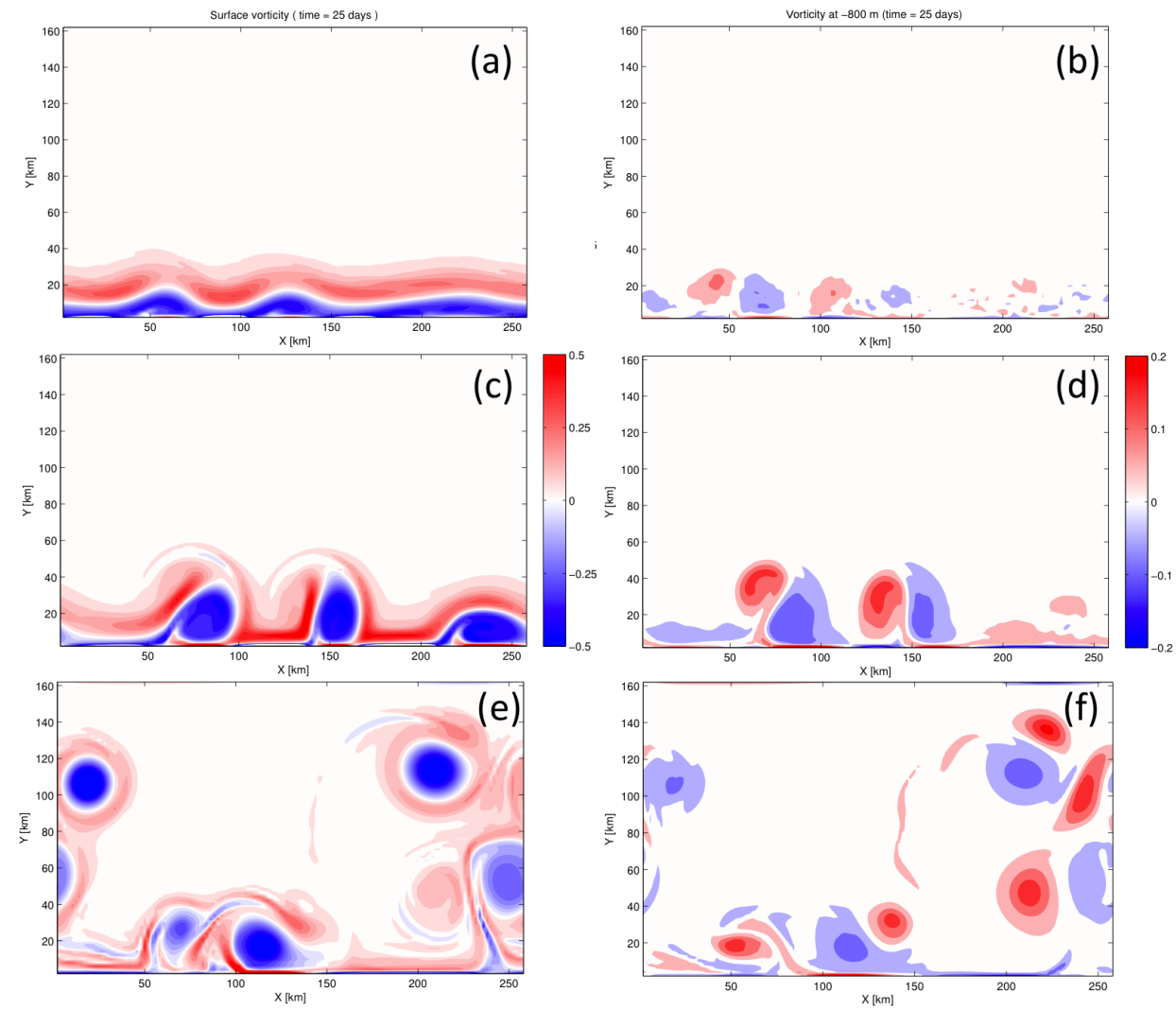

Figure 6. Same as Fig. 5, but for a flat bottom configuration $\left(s=0, T_{\mathrm{p}}=0\right)$ when the aspect ratio parameter is $\gamma=0.24(H=1300 \mathrm{~m})$ and the vortex Rossby number is $R o=0.25$. Panels $(\mathbf{a}, \mathbf{b}),(\mathbf{c}, \mathbf{d})$, and $(\mathbf{e}, \mathbf{f})$ correspond to $t=25, t=35$, and $t=80$ days, respectively.

However, the wave growth does not last long and never until the full breaking. The wave amplitudes get saturated at a level small enough to be hardly competing with the background flow, causing the jet to be barely changing in time (Fig. 5a, c). The flow near the bottom remains very weak both in terms of cross-shore velocity $v^{\prime}$ or vertical velocity $w^{\prime}$ (Fig. 5b, d; note that the colour bar has been magnified by a factor of 5, and the small-scale structures that emerge are due to the initial noise initially introduced at all depths). The kinetic energy source for this ASC regime is the extraction from the horizontal shear, with $\mu_{0}<1$.

A similar non-linear stabilization was found for coastal fronts and currents with the two-layer laboratory experiments performed by Geheniau et al. (2017). The non-linear stabilization curve as a function of the topographic parameter is less abrupt for these physical experiments, but nevertheless, when the ratio of the bottom slope over the isopycnal slopes goes below $T_{0}=s / \alpha<-3$ the non-linear saturation parameter $\varepsilon$ does not exceed 0.1 . Previous studies have shown that bottom topography may have a strong impact on the non-linear saturation of an unstable surface flow. For instance, Sutyrin (2001) showed that, in a Gulf Stream type jet, a very weak bottom slope $s=0.2 \%$ in a deep water case $H=5000 \mathrm{~m}$ can have a negligible impact on the lin- ear growth of the meanders while significantly impacting the non-linear evolution of the unstable current, the eddy formation, and their subsequent shedding.

As stated in Sect. 2, we have also performed a few runs at the higher grid resolution of $\mathrm{d} x=600$. While this change did not significantly affect the growth rate or the wavelength selection of the most unstable modes, it does affect the level of kinetic energy perturbation in this regime (and consequently $\varepsilon$ ). The increased resolution of the cross-shore gradient seems to extend the spectrum of unstable modes, leading to a higher amount of energy in the perturbations. Nevertheless, regardless of the grid resolution, the amplitude of the non-linear saturation parameter $\varepsilon$ always remains weak in this regime.

\section{Coastal meanders and eddy formation}

\subsection{Coastal eddies regime}

The formation of coherent eddies from an unstable coastal current generally results from the pinching off of large meanders. However, these meanders may saturate at an intermediate stage and never lead to the generation of coastal eddies. The value of the non-linear saturation parameter $\varepsilon$ cannot 

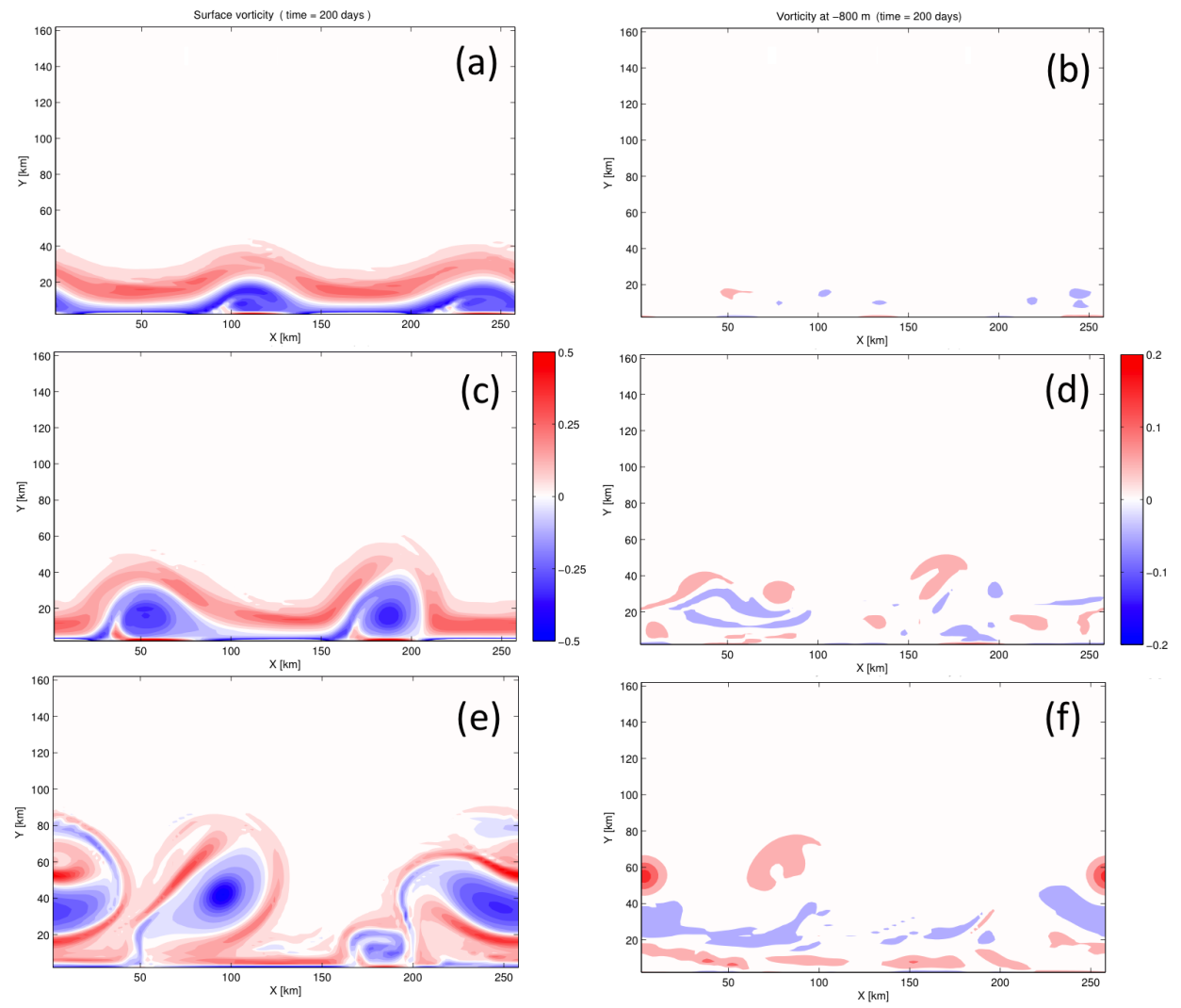

Figure 7. Same as Fig. 5, but for a shelf slope $\left(s=6 \%, T_{\mathrm{p}}=-0.34\right)$ when the aspect ratio parameter is $\gamma \simeq 0.1(H=3000 \mathrm{~m})$ and the vortex Rossby number is $R o=0.25$. Panels $(\mathbf{a}, \mathbf{b}),(\mathbf{c}, \mathbf{d})$, and $(\mathbf{e}, \mathbf{f})$ correspond to $t=200, t=280$, and $t=380$ days, respectively.

distinguish large meanders or coherent eddies, and to perform a quantitative distinction between these two end states we use the vorticity maps. In what follows, we define coherent eddies as vortical structures surrounded with a ring of opposite vorticity, for instance a cyclonic (positive) vorticity ring for an anticyclonic (negative) vorticity core. In other words, when closed contours of zero vorticity appear in the surface vorticity fields, coherent eddies are formed in the upper layer, and we will declare the end state as the coastal eddies regime. In this regime, the eddies systematically detach from the initial location of the coastal current, generating a net cross-shore exchange. As for the buoyant coastal current, the vertical structure of these coherent eddies is baroclinic, with a surface intensification of the vorticity.

However, the vertical structure is not universal and for a similar signature at the surface these coherent eddies could have quite a different structure in the deep layer. Two cases of coastal eddy formation are depicted in Figs. 6 and 7. In the first case, which corresponds to the flat bottom scenarios, the eddies have a signature at depth that is partially in phase with the surface (Fig. 6b, d, f). Dipolar structures are formed in the lower layer with a strong vertical alignment of the anticyclonic cores, consistently with the tendency for barotropization of the flow induced by the standard baroclinic instability.
A similar pattern was described by Gula et al. (2010) in their investigation of the instabilities of a coastal current flowing over a flat bottom in a two-layer shallow water model, suggesting that the vertical structure of the eddies is not significantly altered by the continuous stratification. The anticyclones cores are more intense and robust at the surface, while the cyclonic vorticity is slightly higher than the anticyclonic one in the deep-layer dipoles. We have checked for this specific case that the growth of the kinetic energy of the unstable perturbations is mostly explained by the conversion of potential energy because $\mu_{0}>1$. For the second case, the signature of the growing perturbations in the lower layer vorticity (Fig. 7d, f) is much weaker and the deep-layer velocity almost vanishes even if the surface evolution closely resembles the standard baroclinic instability case. This specific case corresponds to a relatively small vertical aspect ratio $\gamma$ - in other words for deep waters, when baroclinic instability is strongly dampened. Indeed, for this case $\mu_{0}<1$ and the linear stage of instability corresponds to a barotropic shear instability.

Hence, these examples show that two distinct mechanisms of linear instability, namely the baroclinic or the barotropic shear instability, can lead to the same non-linear end state: the formation of coherent eddies in the surface layer which 
are able to trap water mass in their core and escape from the coast. If we consider only the surface signature of coastal eddies, provided by standard remote-sensing measurements such as SST images or SSH maps, we can accurately identify the non-linear coastal eddy regime but hardly make any distinction between the underlying linear instability mechanisms.

\subsection{Coastal meanders}

In contrast to the coastal eddies regime, the formation of coastal meanders corresponds to a non-linear stage where the parameter $\varepsilon$ reaches a finite value (we chose here $\varepsilon \geq 0.2$ ) but coherent eddies are never formed. This is a very interesting non-linear regime for unstable coastal currents that has been, as far as we know, rarely studied. The typical evolution of such coastal meanders is depicted in Fig. 8. The time evolution consists in the growth of the most unstable wave until the amplitude saturates at some level. For instance, in Fig. 8, the non-linear cross-shore energy parameter $\varepsilon$ goes up to 0.74 . The saturation systematically happens before the wave breaks, thus it does not lead to any coherent eddies. The zero-vorticity line, which characterizes the maximal velocity of the initial coastal current, may meander but does not pinch off nor close. After saturation, at longer timescales, other unstable waves have grown enough and start to interact with one another. The overall meander pattern evolves slowly and, in the long term, the flow is dominated by waves travelling along the slope, causing a loss of the initial meander structure. Note that the non-linear interactions of waves is an interesting process in itself, but the wave interactions are probably over-stimulated in these experiments and quite artificial. The reason is that the periodic condition prevents the wave energy radiating away along the topography, and that somehow bounds the waves to a limited domain, forcing them to interact forever.

The vertical structure of such coastal meanders is quite different from the coastal eddies regime. The vorticity is never in phase between surface and bottom, but it is rather in phase quadrature (Fig. 8c, d). The waves have a complex structure in a cross-shore plane (not shown) with several nodal lines but no clear pattern emerging. A key feature of this regime is the bottom intensification of both $v^{\prime}$ and $w^{\prime}$. Such intensification of the cross-shore velocities in the deep layer is often a signature of topographic Rossby waves (see e.g. Huthnance, 1978; Brink, 1991). The $w^{\prime}$ standard deviation ranges from $20 \mathrm{~m}$ to $350 \mathrm{~m} \mathrm{day}^{-1}$, depending on the slope, and reaches its maximum value at the bottom. This bottom intensification would probably be weaker with the presence of bottom friction.

\subsection{A two-parameter space for meanders and coastal eddy formation}

We have shown that $T_{\mathrm{p}}$ is the crucial parameter which controls the final amplitude of the cross-shore perturbations. However, this dimensionless parameter is not the single one that impacts the transition from coherent eddies to coastal meanders. For example, the two distinct dynamical evolutions of the buoyant coastal current depicted in Figs. 7 and 8 correspond to almost equal values of $T_{\mathrm{p}} \simeq-0.33 \pm 0.01$ while another dimensionless parameter, the vertical aspect ratio $\gamma=H_{\text {jet }} /\left(H-H_{\text {jet }}\right)$, differs significantly between these two cases. We found that $\gamma=0.24$ for the coastal meanders in Fig. 7 while $\gamma=0.11$ when coherent eddies are formed (Fig. 6e). Hence, both $T_{\mathrm{p}}$ and $\gamma$ should be taken into account, and we propose a two-parameter space diagram to predict the various non-linear coastal patterns that may be formed from an unstable buoyant current. Figure 9 identifies, in the $\left(T_{\mathrm{p}}, \gamma\right)$ parameter space, the main non-linear regimes between coherent eddies, coastal meanders, and the quasi-stable, alongshore current. The formation of coherent coastal eddies occurs when the topographic parameter or the vertical aspect ratio parameter are small $\left(T_{\mathrm{p}} \gtrsim-0.2\right.$, or $\left.\gamma \lesssim 0.1\right)$, while the emergence of large coastal meanders that remain trapped on the bathymetric slope occurs for a restricted range of values: $\gamma \gtrsim 0.1$ and $-0.4 \lesssim T_{\mathrm{p}} \lesssim-0.2$. Then, for sufficiently negative values of $T_{\mathrm{p}} \lesssim-0.4$ the coastal jet is quasi-stable and the cross-shore perturbations remain asymptotically small.

Note that we have investigated here the range of small and moderate aspect ratio parameter $(\gamma<0.5-0.7)$ which corresponds to a surface-intensified jet. The limit of large $\gamma$ corresponds to deeper jets that extend down to the bottom slope. Such cases were investigated by Isachsen (2011) and Brink (2012). They showed that even if the flow is linearly unstable, the peak of eddy kinetic energy is strongly reduced by an increase in the bottom slope.

\section{Conclusions}

In this paper we have studied the non-linear evolution of an unstable buoyant current, flowing along a coastal slope, for various depths and sloping topographies. The current, kept unchanged, is always linearly unstable. We determined the properties of the linear instability (growth rate, wavelength) from the direct integration of the primitive equations forward in time. The properties of the linear stage (the exponential growth) match published results (Poulin et al., 2014) with, in particular, two unstable branches in the stability diagram: a branch associated with baroclinic instability, where the wavelength of the most unstable mode decreases as slope increases, and a branch associated with horizontal shear instability, where the wavelength is independent of the slope. The advantage of integrating the primitive equations forward in time is to go beyond the linear stage, and to reveal the 

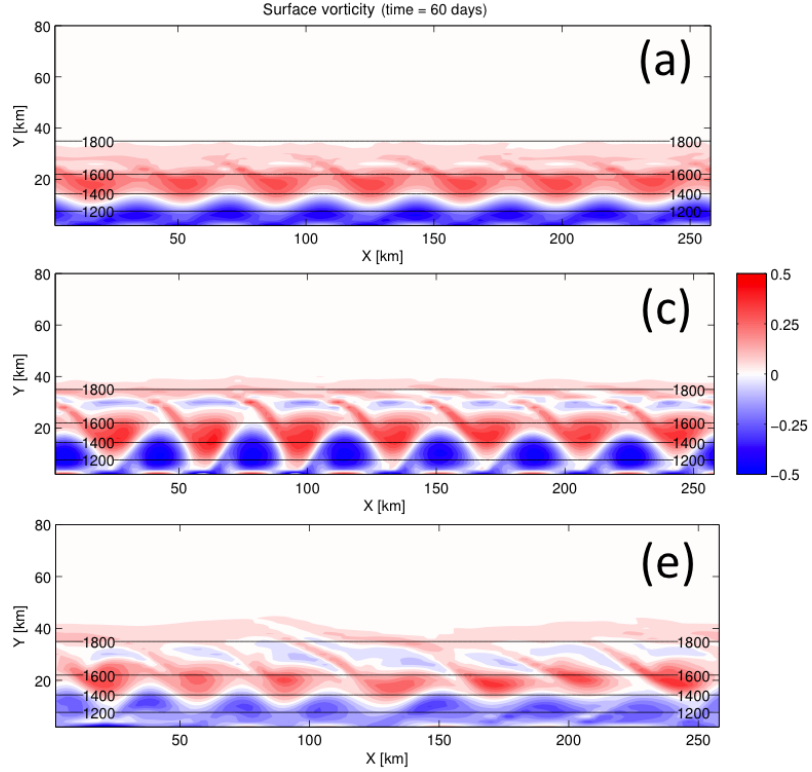
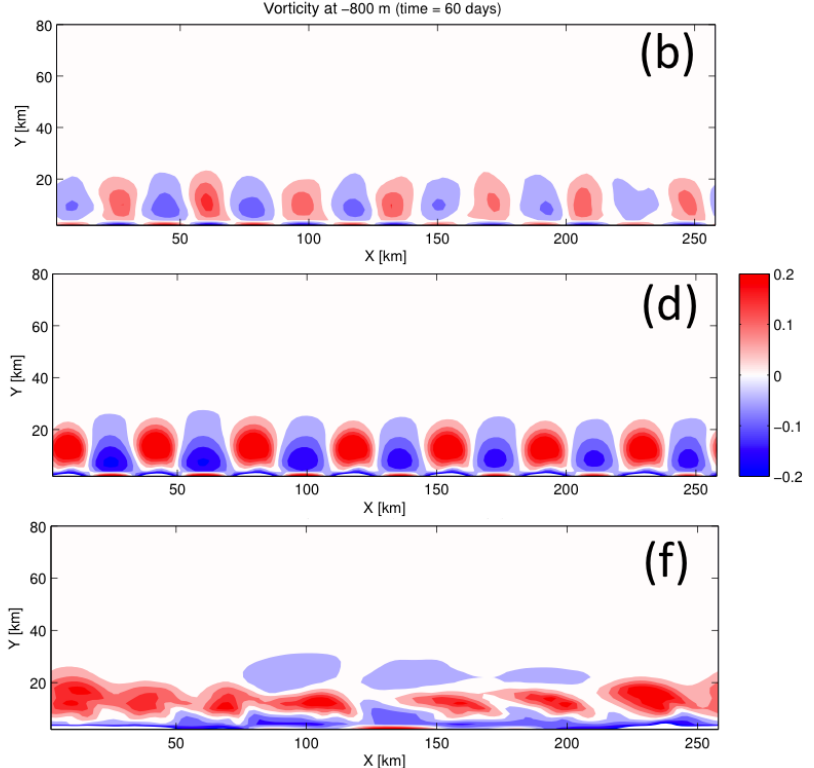

Figure 8. Same as in Fig. 5, but for a shelf slope $\left(s=3 \%, T_{\mathrm{p}}=-0.32\right)$ when the aspect ratio parameter is $\gamma \simeq 0.24(H=1300 \mathrm{~m})$ and the vortex Rossby number is $R o=0.25$. Panels $(\mathbf{a}, \mathbf{b}),(\mathbf{c}, \mathbf{d})$, and $(\mathbf{e}, \mathbf{f})$ correspond to $t=60, t=100$, and $t=150$ days, respectively.

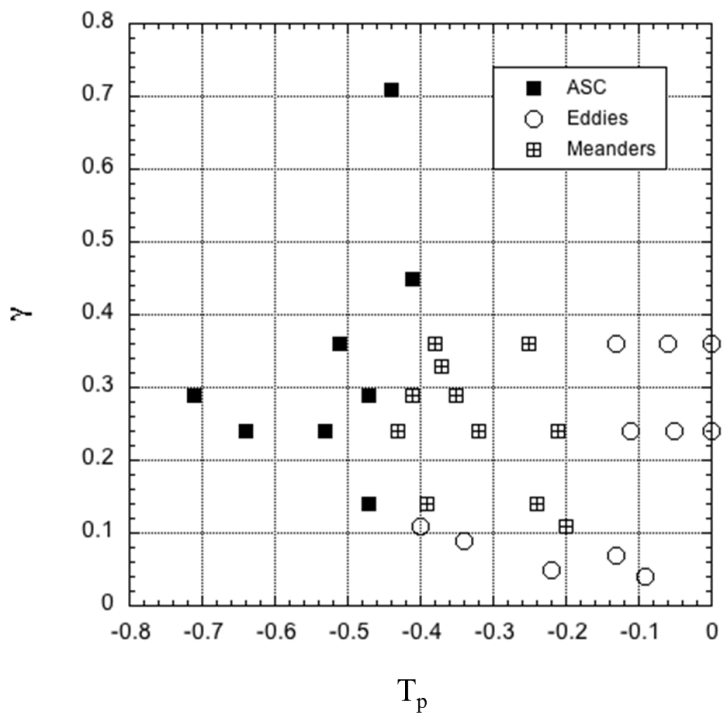

Figure 9. Diagram in the $\left(T_{\mathrm{p}}, \gamma\right)$ parameter space of the various instability regimes: coastal eddies (open circle), coastal meanders (square with cross), and the quasi-stable along-slope current (filled square).

full non-linear evolution. The non-linear regime clearly adds richness and cannot be simply predicted by the linear analysis. Indeed, we found three non-linear end states corresponding to coherent eddies, coastal meanders or quasi-stable jet, while the linear analysis predicted only two types of instability. These distinct non-linear end states correspond to specific patterns that could be easily identified from remote-sensing observations of the ocean surface such as high-resolution SST images. We show in Fig. 10 the typical signature of these three non-linear regimes on the surface temperature field obtained in our simulations (we plot here the results from the high-resolution cases to give a better representation of the SST pattern at meso- and submesoscales). The initial temperature distribution is given by Eq. (3) with a warm along-shore anomaly attached to the coast. The generation of closed patches of warm waters (Fig. 10a) are associated here with coherent eddies, while the undulations of the warm coastal area (Fig. 10b) are associated with the large coastal meanders. In the third regime, the quasi-stable along-shore current, small wavy patterns are visible on the SST front. This in an interesting difference in comparison with the low-resolution $(2 \mathrm{~km})$ cases, because it shows the emergence of submesoscale structures, generated from the initial instability. While these structures are not visible in the low-resolution cases, they never reach a finite amplitude, so their emergence does not change the main results presented in this paper. It confirms that the flow is linearly unstable, but the amplitude of the unstable perturbations hardly affects the along-shore current.

The most interesting finding of this study is that $T_{\mathrm{p}}$ is the key parameter that controls the amplitude of the unstable cross-shore perturbations, defined as the ratio of the crossshore contribution to the kinetic energy to the total kinetic energy. The collapse of experimental points $\left(\varepsilon, T_{\mathrm{p}}\right)$ on a single curve (Fig. 3) is remarkable, given the wide diversity of cases. Moreover, we have checked that complementary points, corresponding to different jet velocities, also collapse on the same curve. This confirms that the ratio $T_{\mathrm{p}}$ of the 

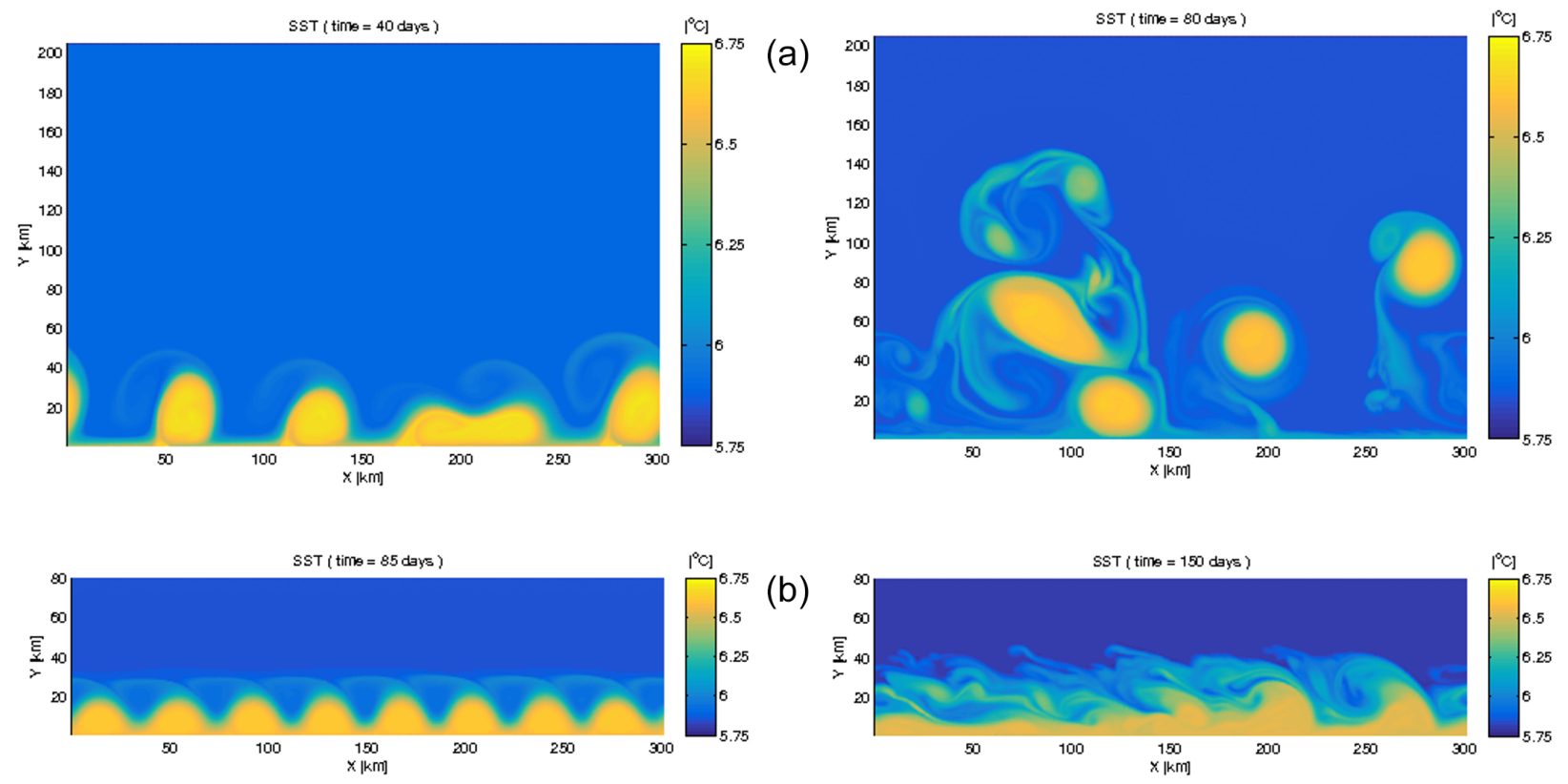

(c)

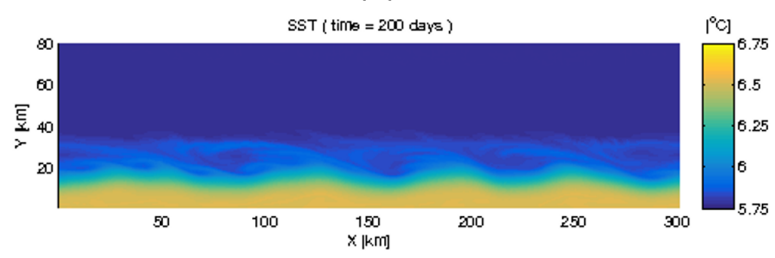

Figure 10. Snapshots of SST for the coastal eddies regime (a), the coastal meanders regime (b), and the quasi-stable along-shore current regime (c). The details of these simulations are presented in Table 2.

Table 2. Same as Table 1, but for the high-resolution cases (600 $\mathrm{m}$ grid resolution).

\begin{tabular}{lrrrrrrrl}
\hline$s(\%)$ & $H$ & $\left|T_{\mathrm{p}}\right|$ & $\gamma$ & $\sigma_{\mathrm{m}} R_{\mathrm{d}} / V_{\max }$ & $\varepsilon$ & $k_{\mathrm{m}} R_{\mathrm{d}}$ & $\mu_{0}$ & Regime \\
\hline 0 & 1300 & 0.00 & 0.24 & 0.027 & 1.28 & 0.52 & 2.44 & eddies \\
3 & 800 & 0.41 & 0.45 & 0.002 & 0.06 & 0.58 & 1.76 & ASC \\
3 & 1300 & 0.32 & 0.24 & 0.012 & 0.74 & 1.03 & 3.08 & meanders \\
\hline
\end{tabular}

topographic Rossby wave phase speed over the jet speed is the generic dimensionless parameter which controls the nonlinear cross-shore patterns of the buoyant coastal current. This parameter is proportional to the topographic slope, and therefore it can be interpreted as a measure of the slope's relative importance. Indeed, even though a slope is dimensionless, it does not adequately quantify whether the topography is steep or gentle in a dynamical sense, while $T_{\mathrm{p}}$ does. For sufficiently negative values of $T_{\mathrm{p}}$ the current is quasi-stable: even if small-scale perturbations could grow, their amplitude would remain small, and thus the mean current follows the along-shore bathymetry and does not lead to any significant cross-shore transport. $T_{\mathrm{p}} \simeq 0.4$ emerges as an important threshold between a quasi-stable, along-shore jet on the one hand, and a non-linear, strongly topographically controlled flow on the other hand.

We have also shown that in addition to the topographic parameter $T_{\mathrm{p}}$, another dimensionless parameter, the vertical aspect ratio of the buoyant current $\gamma=H_{\text {jet }} /\left(H-H_{\text {jet }}\right)$, controls the formation of coherent eddies, which may escape from the coast, or the non-linear meanders, that remain attached to the coast. As far as we know this is the first time that an explicit parameter space is provided for the emergence of coastal eddies or meanders from an unstable buoyant current. This $\left(T_{\mathrm{p}}, \gamma\right)$ parameter space might be quite convenient for real coastal currents because approximated values for these two dimensionless parameters could be easily estimated with a minimal number of in situ mea- 
surements. For instance, hydrographic sections are sufficient to quantify the first baroclinic deformation radius $R_{\mathrm{d}}$, the maximum geostrophic velocity $V_{\max }$, and the vertical extent $H_{\text {jet }}$ of the surface current. Then, a standard bathymetric data set will provide a correct averaging of the bottom slope $s$ in the cross-shore direction. If we consider the coastal Bransfield Current, which inspired the setup of the model used in this study and which has been extensively studied by Savidge and Amft (2009), Sangrá et al. (2011), Poulin et al. (2014), and Sangrá et al. (2017), we get from the combination of numerous CTD stations and ADCP data the following values: $R_{\mathrm{d}} \simeq 10 \mathrm{~km}, U_{\mathrm{max}} \simeq 35 \mathrm{~cm} \mathrm{~s}^{-1}, H_{\text {jet }} \simeq 250 \mathrm{~m}$, and $H \simeq 900-1000 \mathrm{~m}$, while the maximum shelf slope is around $s \simeq 15 \%$ (Poulin et al., 2014). We then obtain $\gamma=0.3-0.4$ and a strongly negative value for the topographic parameter, $T_{\mathrm{p}} \simeq-6$. Therefore, according to our study, the Bransfield Current flows over a very steep shelf slope and should correspond to a quasi-stable, along-shore current. This non-linear analysis extends, to a fully stratified case, the linear stability analysis performed by Poulin et al. (2014) with a twolayer shallow-water model. Such a result is in good agreement with the various drifter paths (Zhou et al., 2002; Poulin et al., 2014), which show that the Bransfield Current does not exhibit any significant meanders along the steep coastal shelf and seems to be relatively stable in the summer months.

This work emphasizes the limitations of linear stability analysis to classify eddy formation, because it does not account for the non-linear saturation which is predominant for large negative $T_{\mathrm{p}}$ values. Nevertheless, we are aware that this work is a first step of a more thorough analysis. The idealized configuration tested here accounts only for initial-value problems, with a weak white noise on the velocity field. This is of course an artificial situation compared to the continually forced circulation in the real ocean. Moreover, the geometry used here may constrain the results in a number of ways. We performed a few tests with different initial velocities and found that there was no effect on the different non-linear end state, but a different jet structure and/or a different distance from the coast are very likely to affect the results, since the initial vertical vorticity would be different. Also, given that the velocity of the topographic Rossby waves is proportional to the width of shelf, this parameter should be also taken into account. The width of the shelf might become particularly important for very steep cases (steeper than the ones tested here), when it becomes comparable with the jet width. Finally, the effect of the bottom friction is here neglected since we focused on the inviscid dynamics and set the friction to zero to prevent a slow-down of the jet during the initial period of integration. The sensitivity of the results presented here to these other parameters will be investigated in a future study.
Data availability. All the simulations performed in this study can be reproduced with the information in the text (domain geometry, resolution, Eqs. (1)-(4), and information in Tables 1 and 2). 
Appendix A: Estimate of the first baroclinic deformation radius

For a continuous stratification $\rho(z)$, the linear eigenmodes and the corresponding deformation radius are given by the equation

$-\partial_{z}\left(\frac{\rho_{0} f^{2}}{g} \frac{\partial_{z} \psi_{n}}{\partial_{z} \rho}\right)=\partial_{z}\left(\frac{f^{2}}{N^{2}(z)} \partial_{z} \psi_{n}\right)=-\frac{\psi_{n}}{R_{\mathrm{d}, n}^{2}}$,

with the appropriate boundary conditions

$$
\begin{array}{ll}
\partial_{z} \psi_{n}=0 & \text { at } z=0 \\
\partial_{z} \psi_{n}=0 & \text { at } z=-H,
\end{array}
$$

where $R_{\mathrm{d}, i}$ are the deformation radius associated to the baroclinic modes $n=1,2, \ldots$. This equation is identical to Eq. (5.204) in Vallis (2006). We plot in Fig. A1 the characteristic density profile obtained in the centre of the coastal jet (Fig. A1a) and an example of the vertical profile of the first baroclinic eigenmode (Fig. A1b).

The first baroclinic deformation radius corresponding to this stratification is $R_{\mathrm{d}, 1}=6.1 \mathrm{~km}$ (this Rossby radius is denoted $R_{\mathrm{d}}$ in the present study). This value is smaller than the typical width of the jet $L=10 \mathrm{~km}$. According to Fig. A1c the Rossby radius $R_{\mathrm{d}}$ increases with the water depth $H$. In other words, the Burger number $B u$ decays when the aspect ratio parameter $\gamma$ increases. For the range of parameters used in this study the Burger number remains small. 

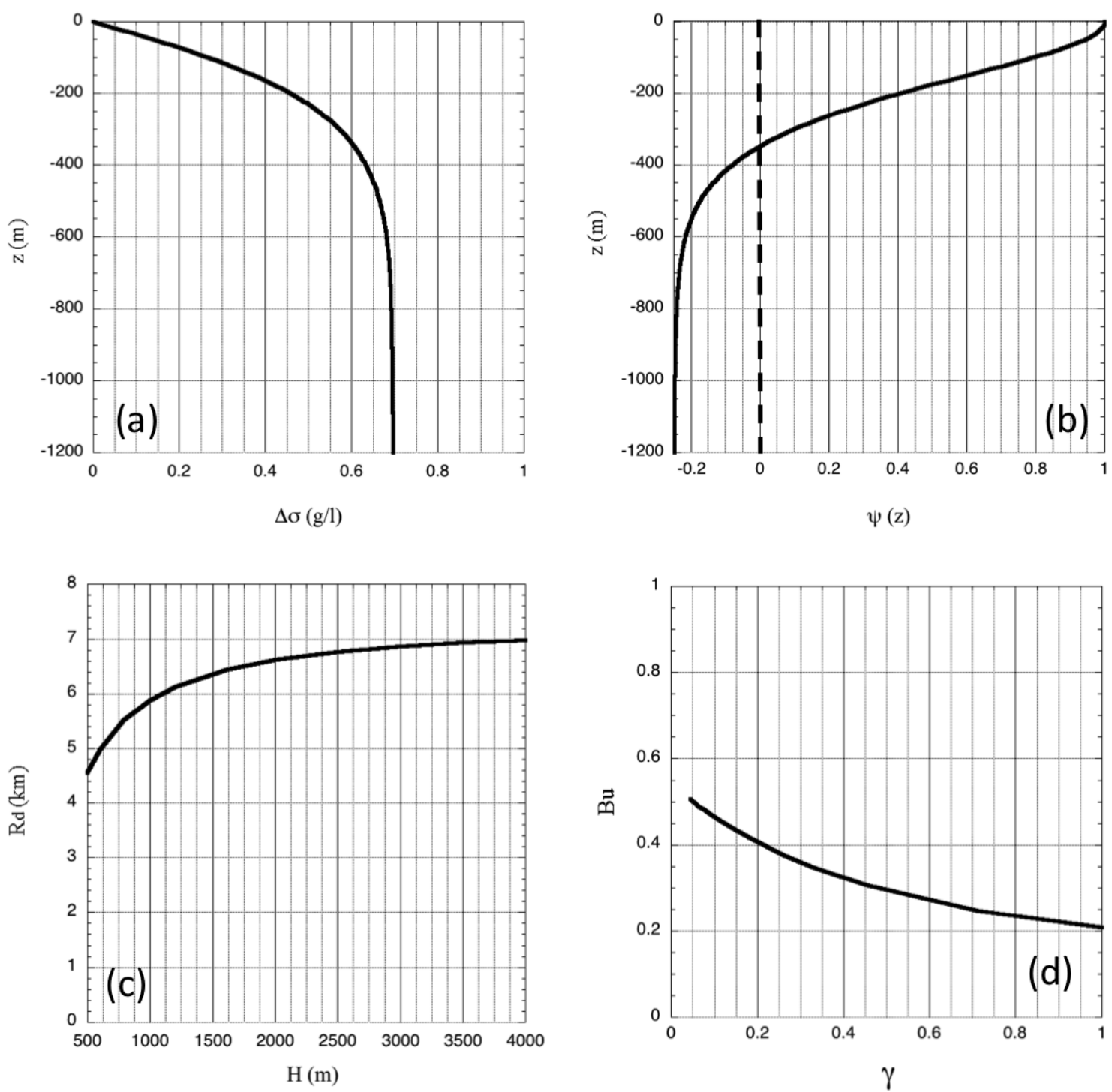

Figure A1. Vertical profile of the potential density anomaly $\Delta \sigma(z)$ (a) and the vertical structure of the first baroclinic eigenmode (b) computed in the centre of the coastal current $y=D$ for a water depth $H=1200 \mathrm{~m}$. The evolution of the first baroclinic deformation radius $R_{\mathrm{d}}$ is plotted in panel (c) as a function of the water depth $H$. Panel (d) shows the evolution of the corresponding Burger number $B u=\left(R_{\mathrm{d}} / L\right)^{2}$ as a function of $\gamma=H_{\text {jet }} /\left(H-H_{\text {jet }}\right)$. 
Competing interests. The authors declare that they have no conflict of interest.

Acknowledgements. We acknowledge helpful discussions with Andrew Stewart, and the anonymous reviewers for their useful comments. This work was funded by the ANR Astrid Project SYNBIOS (ANR 11 ASTR 014 01).

Edited by: John M. Huthnance

Reviewed by: two anonymous referees

\section{References}

Birol, F., Cancet, M., and Estournel, C.: Aspects of the seasonal variability of the Northern Current (NW Mediterranean Sea) observed by altimetry, J. Marine Syst., 81, 297-311, 2010.

Björk, G., Gustafsson, B., and Stigebrandt, A.: Upper layer circulation of the Nordic seas as inferred from the spatial distribution of heat and freshwater content and potential energy, Polar Res., 20, 161-168, 2001.

Blumsack, S. and Gierasch, P.: Mars - The effects of topography on baroclinic instability, J. Atmos. Sci., 29, 1081-1089, 1972.

Brink, K. H.: Coastal-trapped waves and wind-driven currents over the continental shelf, Annu. Rev. Fluid. Mech., 23, 389-412, 1991.

Brink, K. H.: Baroclinic instability of an idealized tidal mixing front, J. Mar. Res., 70, 661-688, 2012.

Debreu, L., Marchesiello, P., Penven, P., and Cambon, G.: Two-way nesting in split-explicit ocean models: algorithms, implementation and validation, Ocean Model., 49-50, 1-21, 2011.

Eden, C. and Boning, C.: Sources of eddy kinetic energy in the Labrador Sea, J. Phys. Oceanogr., 32, 3346-3363, 2002.

Geheniau, N., Pennel, R., and Stegner, A.: Non-linear stabilization of coastal fronts and currents by a sloping bathymetry: laboratory experiments, in preparation, 2017.

Gula, J. and Zeitlin, V.: Instabilities of shallow-water flows with vertical shear in the rotating annulus. Modelling Atmospheric and Oceanic flows: insights from laboratory experiments and numerical simulations, Amer. Geophys. Union, edited by: von Larcher, T. and Williams, P., ISBN 978-1-118-85593-5, 2014.

Gula, J., Zeitlin, V., and Bouchut, F.: Instabilities of buoyancydriven coastal currents and their nonlinear evolution in the twolayer rotating shallow water model. Part 2. Active lower layer, J. Fluid Mech., 665, 209-237, 2010.

Gula, J., Molemaker, M., and McWilliams, J.: Submesoscale cold filaments in the Gulf Stream, J. Phys. Oceanogr., 44, 2617-2643, 2014.

Gula, J., Molemaker, M., and McWilliams, J.: Gulf Stream dynamics along the southeastern US seaboard, J. Phys. Oceanogr., 45, 690-715, 2015.

Hátún, H., Eriksen, C., and Rhines, P.: Buoyant eddies entering the Labrador Sea observed with gliders and altimetry, J. Phys. Oceanogr., 37, 2838-2854, 2007.

Huthnance, J.: On coastal trapped waves: analysis and numerical calculation by inverse iteration, J. Phys. Oceanogr., 8, 74-92, 1978.
Isachsen, P. A.: Baroclinic instability and eddy tracer transport across sloping bottom topography: how well does a modified Eady model do in primitive equation simulations?, Ocean Model., 39, 183-199, 2011.

Knox, J. A.: Inertial instability, edited by: Holton, J. R., Curry, J. A., and Pyle, J. A., Encycl. Atmos. Sci., 1004-1013, 2003.

Lozier, M. and Reed, M.: The influence of topography on the stability of shelfbreak fronts, J. Phys. Oceanogr., 35, 1023-1036, 2005.

Lozier, M., Reed, M., and Gawarkiewicz, G.: Instability of a shelfbreak front, J. Phys. Oceanogr., 32, 924-944, 2002.

Mechoso, C.: Baroclinic instability of flows along sloping boundaries, J. Atmos. Sci., 37, 1393-1399, 1980.

Millot, C.: Circulation in the western Mediterranean Sea, J. Marine Syst., 20, 423-442, 1999.

Mysak, L.: On the stability of the California Undercurrent off Vancouver Island, J. Phys. Oceanogr., 7, 904-917, 1977.

Mysak, L., Muench, R. D., and Schumacher, J. D.: Baroclinic Instability in a downstream varying channel: Shelikof Strait, Alaska, J. Phys. Oceanogr., 11, 950-969, 1981.

Obaton, D., Millot, C., d'Hières I., G. C., and Taupier-Letage: The Algerian Current: comparisons between in situ and laboratory data sets, Deep-Sea Res., 47, 2159-2190, 2000.

Pedlosky, J.: Geophysical Physical Dynamics, Springer New York, 1987.

Pennel, R., Stegner, A., and Béranger, K.: Shelf impact on buoyant coastal current instabilities, J. Phys. Oceanogr, 42, 39-61, 2012.

Penven, P., Debreu, L., Marchesiello, P., and McWilliams, J. C.: Evaluation and application of the ROMS 1-way embedding procedure to the central california upwelling system, Ocean Model., 12, 157-187, 2006.

Phillips, N. A.: Energy transformations and meridional circulation associated with baroclinic waves in a two-level, quasigeostrophic model, Tellus, 6, 273-286, 1954.

Pickart, R. S., Torres, D., and Fratantoni, P.: The East Greenland Spill Jet, J. Phys. Oceanogr., 35, 1037-1053, 2005.

Plougonven, R. and Zeitlin, V.: Nonlinear development of inertial instability in a barotropic shear, Phys. Fluids, 21, 106601, 2009.

Poulin, F. and Flierl, G.: The influence of topography on the stability of jets, J. Phys. Oceanogr., 35, 811-825, 2005.

Poulin, F., Stegner, A., Arencibia, M. H., Diaz, A. M., and Sangrá, P.: Steep Shelf Stabilization of the Coastal Bransfield Current: Linear Stability Analysis, J. Phys. Oceanogr., 44, 714-732, 2014.

Puillat, I., Taupier-Letage, I., and Millot, C.: Algerian eddies lifetime can near 3 years, J. Marine Syst., 31, 245-259, 2002.

Riandey, V., Champalbert, G., Carlotti, F., Taupier-Letage, I., and Thibault-Botha, D.: Zooplankton distribution related to the hydrodynamic features in the Algerian Basin (western Mediterranean Sea) in summer 1997, Deep-Sea Res., 52, 2029-2048, 2005.

Sammari, C., Millot, C., and Prieur, L.: Aspects of the seasonal and mesoscale variabilities of the Northern Current in the western Mediterranean Sea inferred from the PROLIG-2 and PROS-6 experiments, Deep-Sea Res. Pt. I, 42, 893-917, 1995.

Sangrá, P., Gordo, C., Arencibia, M. H., Marrero-Díaz, A., Rodríguez-Santana, A., Stegner, A., Martínez-Marrero, A., Pelegrí, J. L., and Pichon, T.: The Bransfield Current System, DeepSea Res., 58, 390-402, 2011. 
Sangrà, P., Stegner, A., Hernández-Arencibia, M., Marrero-Díaz, Á., Salinas, C., Aguiar-González, B., Henriquez-Pastene C., and Mouriño-Carballido, B.: The Bransfield gravity current, DeepSea Res. Pt. I, 119, 1-15, 2017

Savidge, D. and Amft, J.: Circulation on the west Antarctic Peninsula derived from 6 years of shipboard ADCP transects, DeepSea Res., 56, 1633-1655, 2009.

Shchepetkin, A. F. and McWilliams, J. C.: A method for computing horizontal pressure-gradient force in an oceanic model with a nonaligned vertical coordinate, J. Geophys. Res., 108, 19782012, 2003.

Shchepetkin, A. F. and McWilliams, J. C.: The regional oceanic modeling system (roms): a split-explicit, free-surface, topography-following-coordinate oceanic model, Ocean Model., 9, 347-404, 2005.
Stewart, A. L. and Thompson, A. F.: Connecting Antarctic crossslope exchange with Southern Ocean overturning, J. Phys. Oceanogr., 43, 1453-1471, 2013.

Sutyrin, G.: Effects of a topographic slope on the $\beta$-drift of a baroclinic vortex, J. Mar. Res., 59, 977-989, 2001.

Vallis, G. K.: Atmospheric and Oceanic Fluid Dynamics, Cambridge University Press, Cambridge, UK, 2006.

Williams, P. and Kelsall, C.: The dynamics of baroclinic zonal jets, J. Atmos. Sci., 72, 1137-1151, 2015.

Zhou, M., Niiler, P., and Hu, J.: Surface currents in the Bransfield and Gerlache Straits, Antarctica, Deep-Sea Res., 49, 267-280, 2002. 